\title{
La importancia de las interacciones entre vecinos con lazos sociales débiles para incrementar la participación laboral femenina en Chile
}

\author{
Bárbara Flores \\ Universidad de Chile, Chile
}

\begin{abstract}
RESUMEN: Este artículo estudia factores culturales que podrían explicar las bajas tasas de participación laboral femenina (PLF) observadas en Chile. Se estima un modelo de elección discreta con interacciones sociales, incluyendo como factor explicativo de la participación laboral individual, la tasa de participación laboral de las mujeres que viven en el mismo barrio o zona censal. Para ello, se construye una base de datos inédita que vincula las tasas de PLF a nivel de zonas censales, extraídas del Censo 2017, a las mujeres encuestadas en el Estudio Longitudinal Social de Chile (ELSOC). Luego, para analizar la heterogeneidad de los resultados, se construye un indicador de fortaleza de lazos sociales conformados en el barrio y el modelo se estima para mujeres
\end{abstract}

BÁRbARA Flores es Doctora en Economía, University College London, Reino Unido. Investigadora posdoctoral asociada al Centro de Estudios de Conflicto y Cohesión Social (COES) y al Núcleo Milenio en Desarrollo Social (DESOC), del Departamento de Economía, Universidad de Chile. Dirección: Diagonal Paraguay 257, torre 26, of. 1501, Santiago Centro, Santiago, Chile, CP 8330015. Email: barflore@fen.uchile.cl.

Este trabajo fue financiado por ANID/FONDAP/15130009 y ANID, Programa Iniciativa Científica Milenio, Código NCS17_015. La autora agradece al equipo de la Encuesta Longitudinal Social de Chile (ELSOC) de COES por otorgar el acceso a los datos y por vincular información territorial proveniente de otras fuentes. Asimismo, agradece los enriquecedores comentarios de Matías Berthelon, Sebastián Gallegos, Esteban Puentes, Quentin Ramond, Claudia Sanhueza, Javiera Selman, y a los asistentes a diversos seminarios internos de COES, DESOC y de la Escuela de Negocios de la Universidad Adolfo Ibáñez, Chile. Delia Moens De Hase y Sofía Bravo realizaron un excelente trabajo como ayudantes de investigación. 
con lazos débiles y fuertes, por separado. Los resultados indican que las interacciones sociales a nivel de barrio están relacionadas de manera positiva y estadísticamente significativa con la decisión individual de participar en el mercado del trabajo solo para aquellas mujeres de baja escolaridad con lazos débiles en el barrio de residencia. Esto es consistente con la teoría, ya que los lazos débiles actúan como puentes hacia nuevas fuentes de información y redes sociales que pueden ser relevantes para participar en el mercado laboral. Estos resultados son importantes porque la existencia de estos efectos sociales implica un efecto multiplicador que puede ser utilizado por la política pública para amplificar el efecto de programas a pequeña escala, especialmente en barrios con altos índices de vulnerabilidad socioeconómica.

PALABRAS CLAVE: oferta laboral, participación laboral femenina, interacciones sociales, lazos sociales, cohesión social

RECIBIDO: diciembre 2020 / ACEPTADO: mayo 2021

\section{The Role of Social Interactions among Weak-Tied Neighbors to Increase Female Labor Force Participation in Chile}

ABstRACT: This article studies cultural factors that could explain the actual low rates of female labor force participation (FLP) in Chile. A discrete choice model with social interactions is estimated by including the female neighbors' labor force participation rate as a regressor in the model. To do so, a unique dataset is constructed by merging the FLP rates at the census zone level from the Chilean Census 2017 to the surveyed women in the Chilean Longitudinal Social Study (ELSOC). In order to analyze the heterogeneity of the results, an index of the strength of the ties within neighbors is constructed and the model is estimated separately for women with weak and strong ties within the neighborhood. The results indicate a significant and positive correlation between neighbors' FLP rate and the individual decision of participation in work only for low-skilled women who have weak ties within the neighborhood of residence. This is consistent with the theory, as weak ties act as a bridge between low-skilled women and new information and social networks that could be relevant to participate in the labor market. These results are relevant as the existence of the social effect implies a social multiplier that can be used for policy to expand the effect of programs of small scale, especially in neighborhoods with high socioeconomic vulnerability indexes.

KEYWORDS: labor supply, female labor force participation, social interactions, social ties, social cohesion

ReCEIVED: December 2020 / Accepted: May 2021 
nivel mundial, la tasa de participación laboral femenina, definida
como la proporción de mujeres mayores de 15 años que trabaja o busca trabajo con respecto al total de mujeres en el mismo tramo de edad, ha aumentado de manera persistente durante los últimos 50 años. Sin embargo, en la mayoría de los países sigue existiendo una marcada brecha de género en las tasas de participación laboral (Cahuc, Carcillo y Zylberberg 2014). A nivel macro, este hecho tiene relevantes implicancias para las economías, ya que existe una relación positiva entre desarrollo y participación laboral femenina (Duflo 2012). A nivel micro, la inclusión de la mujer en el mercado del trabajo incrementa los ingresos del hogar, constituye un mecanismo para la superación de la pobreza y mantención fuera de esta. Adicionalmente, la participación de mujeres en el mercado laboral incrementa su empoderamiento económico, social y político (Bali y Wallentin 2012; Goldin 2006; Kabeer 2018).

Los países han desarrollado estrategias para mejorar la participación laboral femenina, promover el desarrollo económico y disminuir desigualdades de género, basados en la teoría clásica del capital humano (Mincer 1962; Behrman y Wolfe 1984; Killingsworth y Heckman 1986; Blundell, Ham y Meghir 1987). En particular, el incremento en los años de escolaridad obligatorios, métodos anticonceptivos, provisión de cuidado infantil, capacitación y horarios laborales flexibles, son algunas políticas que han sido implementadas para promover la inserción de la mujer en el mercado del trabajo.

Recientemente, se ha internalizado la necesidad de incluir factores sociales en los modelos tradicionales de capital humano. Las normas sociales y culturales en una sociedad también pueden influir en las tasas de participación laboral (Codazzi, Pero y Albuquerque 2018; Contreras y Plaza 2010; Chen y Ge 2018; Kabeer 2020). También, los modelos económicos han comenzado a reconocer que los individuos forman parte de una red social, cuyas acciones y reacciones pueden modificar su comportamiento individual. Estas interdependencias directas entre las decisiones de los individuos, que no están mediadas por mercados o contratos, se denominan interacciones sociales (Brock y Durlauf 2001b).

Sin embargo, el caso específico de interacciones sociales entre mujeres y sus resultados en el mercado laboral, ha sido poco explorado (Mota et al. 2016; Maurin y Moschion 2009; Nicoletti, Salvanes y Tominey 2018). Expandir esta evidencia es relevante porque las interacciones so- 
ciales pueden crear externalidades y amplificar el efecto de programas a través de un multiplicador social (Manski 1993; Durlauf 2004). Asimismo, resulta importante analizar no solo la existencia de las interacciones sociales y su efecto sobre la decisión individual de las mujeres, sino también la intensidad de dichas interacciones. En particular, las características de los lazos sociales que construyen las mujeres, también pueden influir en su decisión individual de integrarse al mercado del trabajo. De acuerdo a Granovetter (1973), existen lazos fuertes que corresponden al círculo más cercano y de confianza. Pero también destaca la importancia de los lazos débiles, porque ellos permiten la creación de puentes hacia otras redes sociales y el acceso a información que no existirían si las noticias y opiniones se limitaran solo a aquellas provistas por los lazos fuertes.

Este artículo contribuye a esta línea de investigación, combinando el análisis de las interacciones sociales entre mujeres y el tipo de lazos sociales con que cuentan dentro de un mismo barrio. Específicamente, el objetivo de esta investigación es determinar si la probabilidad de participar en el mercado laboral está relacionada con las interacciones sociales que experimentan las mujeres dentro de su barrio de residencia. Luego, se explora la heterogeneidad de los resultados, según fortaleza o debilidad de los lazos que tiene cada mujer en su vecindario.

Se analiza el caso de Chile, un caso especial dentro de los países de América Latina. Si bien este país exhibe altos índices relativos de educación y crecimiento, presenta una tasa de participación laboral femenina de 49\% (MDS 2017), menor al 52\% de la región (CEPAL 2019) y muy por debajo del $65 \%$ de países desarrollados (OECD 2020). Este fenómeno se conoce como la excepción chilena y ha sido destacado por Contreras, Hurtado y Sara (2012) y por McKeown, Haji y Ferguson (2016). Esto, porque se han implementado programas que podrían incrementar las tasas de participación femenina en el país, a través de la provisión de cuidado infantil (Bentancor 2013; Medrano 2009; Encina y Martínez 2009), extensión de la jornada escolar (Contreras y Sepúlveda 2017; Martínez y Perticará 2017), más educación (Larrañaga 2006) o programas de empleo (Carneiro, Galasso y Ginja 2013, 2019; Piras y Rucci 2014), obteniendo nulos o pequeños resultados esperados. Esta evidencia indica que existen factores sociales específicos que inciden en la participación laboral de las mujeres y que aún deben ser explicados. 
Además, se estudian interacciones sociales a nivel de barrios porque: (i) en estos ocurren interacciones sociales de manera natural y periódica; (ii) permiten ubicar social y espacialmente a las mujeres, y (iii) las tasas de participación laboral femenina no se distribuyen de manera aleatoria en el territorio. Asimismo, el foco se establece en mujeres de baja escolaridad, que tienen hasta educación secundaria completa, porque las tasas de participación laboral de mujeres con educación terciaria y de posgrado alcanzan el promedio de países desarrollados, al igual que en el caso de los hombres (MDS 2017). Evidencia previa sugiere que la interacción entre vecinas disminuye a medida que aumenta la educación y el nivel de ingresos, debido a que las redes sociales relevantes no se limitan al espacio geográfico. En contraste, en sectores de escasos recursos las interacciones son principalmente locales (García, Carrasco y Rojas 2014; Holanda 2000; Marques, Moya y Zoppi 2008). Este artículo muestra que la decisión de participación laboral de hombres y mujeres con altos niveles de educación no está correlacionada con las decisiones laborales de los vecinos o vecinas.

De manera general, las interacciones sociales pueden influir sobre decisiones individuales a causa de tres factores principales. Primero, factores psicológicos como la inspiración o la identidad, que inducirín a las mujeres a participar en el trabajo, tal como lo hacen otras mujeres (Akerlof y Kranton 2000). Segundo, interdependencias en las restricciones que enfrentan las mujeres pueden afectar los costos de participar en el mercado laboral cuando otras mujeres están trabajando (Manski 2000). Tercero, la transmisión de información podría afectar las percepciones sobre las oportunidades en el mercado laboral y las ventajas del trabajo (Durlauf 2004).

En la práctica, esta investigación se basa en la literatura de elección discreta con interacciones sociales (Brock y Durlauf 2001a) y extiende un modelo estándar de participación laboral femenina (Blundell y Powell 2004). En este, una mujer decide si participa o no en el mercado del trabajo en función de sus características sociodemográficas; se incluye como factor explicativo la tasa de participación laboral de las mujeres que residen en el mismo barrio, definido como zona censal. Si bien esta definición geográfica consiste en una división administrativa del país, se ajusta a lo que las personas consideran como su barrio en zonas urbanas (Link y Valenzuela 2018). De acuerdo a este modelo, si el coeficiente estimado es mayor que cero, reflejaría una complementariedad estratégica 
entre las elecciones de las mujeres, implicando que, al decidir si trabajan o no, se ven influidas positivamente por las vecinas que participan.

Con datos del Censo 2017 del Instituto Nacional de Estadísticas (INE), se vincula la tasa de participación laboral femenina de la zona censal de residencia a las mujeres encuestadas en el Estudio Longitudinal Social de Chile (ELSOC) del Centro de Estudios de Conflicto y Cohesión Social (COES 2017) en su segunda ronda, el cual contiene rica información respecto de las características de cada mujer, su estatus laboral y su barrio de residencia. Esta vinculación de datos es inédita y permite contar con características del vecindario de residencia para cada mujer encuestada. Pocos estudios han utilizado la proximidad geográfica para estudiar la influencia de las interacciones sociales en el comportamiento en el mercado laboral (para Estados Unidos: Bayer, Ross y Topa 2008; Mota et al. 2016; Schmutte 2015; para Inglaterra: Guerra y Mohnen 2014; para Francia: Maurin y Moschion 2009; para Noruega: Nicoletti, Salvanes y Tominey 2018).

Pero no solo la presencia de interacciones sociales es relevante, sino también el tipo de lazo conformado entre las mujeres y los vecinos con quienes ellas interactúan. Por lo tanto, se construye un indicador de fortaleza de lazos sociales, basado en la definición de Granovetter (1973). En su influyente artículo sobre la importancia de los lazos débiles, el autor indica que la fortaleza de un lazo entre individuos depende del tiempo que comparten, la intensidad emocional del vínculo, la intimidad y confianza mutua, y la reciprocidad de la relación. Los datos utilizados permiten generar indicadores que son proxys de estas dimensiones y consolidarlos en un indicador global. Este indicador es utilizado para analizar la heterogeneidad de las correlaciones estimadas en el modelo empírico, según el tipo de lazo social que tiene cada mujer con sus vecinos. Para ello se estima el modelo por separado para las mujeres que tienen lazos débiles y luego, para aquellas con lazos fuertes en el vecindario.

Los resultados indican que las interacciones sociales a nivel de barrio están asociadas de manera significativa y positiva con la decisión individual de participar en la fuerza laboral, solo para aquellas mujeres con lazos débiles en el barrio de residencia. La estimación del modelo indica que el coeficiente asociado a la tasa de participación laboral de las vecinas cercanas es estadísticamente significativo y distinto de cero. Específicamente, un aumento de una desviación estándar en la participa- 
ción laboral femenina del barrio de residencia está asociado a un aumento promedio de 8 puntos porcentuales en la probabilidad individual de participar en el mercado del trabajo. Para mujeres con lazos fuertes en el vecindario, esta asociación no es estadísticamente significativa.

Este resultado es consistente con la teoría de Granovetter (1973), quien indicaba que aquellos lazos débiles conformados entre personas son los que generan puentes hacia información nueva, difícil de acceder a través de lazos fuertes por pertenecer a otra red de contactos. Como implicancia de política pública, se podría explotar el efecto multiplicador proveniente de las interacciones sociales entre vecinas en zonas con mayor vulnerabilidad socioeconómica, generando instancias locales de diálogo y colaboración entre mujeres que participan en el mercado laboral y aquellas que no.

Cabe señalar que los resultados no necesariamente representan un efecto causal. En efecto, existen desafíos para identificar los efectos de las interacciones sociales. Primero, hay un problema de simultaneidad debido a que existen movimientos simultáneos en las decisiones y resultados obtenidos por las mujeres (Manski 1993). Segundo, shocks no observables y el entorno institucional del vecindario podrían generar correlaciones en atributos no observados. Por lo tanto, comovimientos en las decisiones de mujeres del mismo barrio pueden deberse a la presencia de factores no observados correlacionados a nivel de vecindario más que a la presencia de interacciones sociales (Topa 2011). Tercero, los individuos se distribuyen en los vecindarios en función de sus características y también porque tienen preferencias similares (Topa 2001).

Por lo tanto, los resultados que expone este artículo deben interpretarse con precaución. Las correlaciones estimadas corresponden a un análisis descriptivo de la asociación empírica que existe entre interacciones sociales a nivel de barrio y la decisión individual de integrarse al mercado laboral por parte de las mujeres de baja escolaridad y con lazos débiles en el vecindario. Dada la escasa evidencia actualmente disponible respecto de este tema, este artículo puede orientar estudios posteriores que avancen en la identificación del efecto causal, en un contexto de tasas de participación laboral femenina particularmente bajas, como es Chile. Es así como, hacia el final del artículo, se plantean posibles direcciones para futuras investigaciones. 
El resto del artículo está organizado de la siguiente manera. La sección 1 analiza la importancia del barrio para las interacciones y conformación de lazos sociales. La sección 2 describe el modelo teórico y presenta la aplicación empírica. La sección 3 describe los datos y presenta estadísticas descriptivas de la muestra de mujeres bajo análisis. La sección 4 contiene los resultados de la estimación. Finalmente, la sección 5 concluye y sugiere estrategias de identificación del efecto causal para estudios futuros. Adicionalmente, se presentan cinco anexos con material complementario que incluyen figuras, tablas y demostraciones para profundizar en ciertos argumentos del texto principal.

\section{EI barrio y las interacciones sociales}

\section{La importancia del barrio}

Conceptualmente, existen diversas definiciones de barrio provenientes desde el urbanismo. En general, los autores concuerdan en que un barrio corresponde a una unidad territorial que posee una identidad específica, en cuanto a morfología y fisionomía, que lo distingue de otras unidades dentro de la ciudad (Tapia, Lange y Larenas 2009). Sin embargo, su delimitación presenta dificultades, ya que puede responder a una división administrativa, funcional o por áreas homogéneas, entre otras (Tapia 2013).

Estudios basados en análisis territoriales a escala barrial han incrementado su popularidad (Atkinson, Dowling y McGuirk 2009; Andersson y Musterd 2005, 2010), principalmente porque las políticas recientes alrededor del mundo han adoptado un enfoque de barrio, constituyendo un elemento estratégico para identificar problemáticas específicas, vulnerabilidades, focalizar recursos e implementar programas (Tapia 2013; Kearns y Parkinson 2001).

En este artículo se analizan las interacciones sociales que ocurren dentro de las zonas censales de Chile, ${ }^{1}$ las cuales corresponden a conglomerados de manzanas en el área urbana del país, identificados por

\footnotetext{
${ }^{1}$ División geográfica censal: el territorio comunal se divide en distritos urbanos, rurales o mixtos. En el área urbana se identifican zonas censales compuestas de manzanas y en el área rural, localidades compuestas de entidades. Los límites los define el INE y se encuentran enmarcados en los límites de la división político-administrativa. Sin embargo, su trazado no reviste un carácter legal, solo responde a criterios logísticos para el levantamiento de datos (INE 2019).
} 
el INE. ${ }^{2}$ Según Link y Valenzuela (2018), su extensión coincide con la percepción de barrio que tienen sus residentes y se ha validado como tal en la investigación urbana. Por lo tanto, para efectos de los siguientes análisis, un barrio corresponde a una zona censal.

Figura 1. TASA DE PARTICIPACIÓN LABORAL FEMENINA SEGÚN ZONAS CENSALES. REGIÓN METROPOLITANA, AÑO 2017

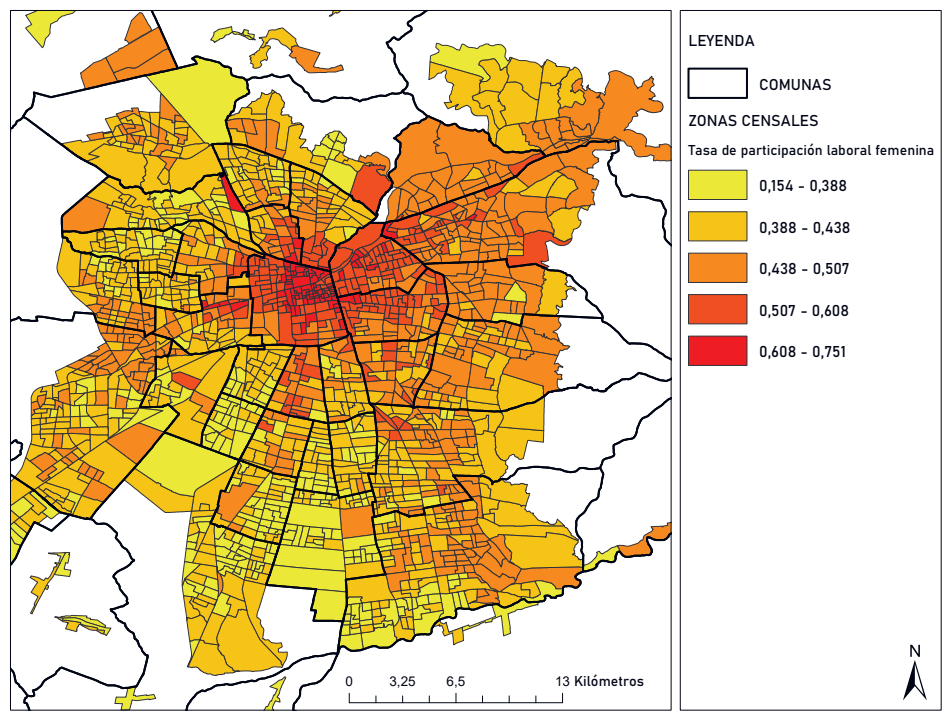

Fuente: Elaboración propia en base a Censo 2017, INE.

Específicamente, el interés por estudiar interacciones sociales a nivel de barrio surge por la constatación empírica de una distribución no aleatoria de las tasas de participación laboral femenina a lo largo del territorio. Para ilustrar esta afirmación, la Figura 1 presenta las tasas de participación femenina según zona censal en la Región Metropolitana. En este mapa se diferencian por color, cinco tramos de participación. Las zonas en rojo exhiben las tasas de participación más altas, similares al promedio del 65\% de países desarrollados (OECD 2020) y se encuentran en comunas tradicionalmente asociadas a mayores niveles de ingreso y escolaridad. En contraste, las menores tasas de participación, en color

\footnotetext{
2 El área promedio de una zona censal (urbana) es de 0,8 kilómetros cuadrados y la mediana es de 0,5 kilómetros cuadrados. Si la zona censal tipo fuera un cuadrado, tendría un lado de longitud de 0,7 kilómetros y una distancia del centro al vértice de 0,4 kilómetros (unas 4 cuadras, aproximadamente). Cálculos propios en base a cartografía digital del INE.
} 
amarillo, se encuentran en zonas periféricas, generalmente asociadas a menores niveles de ingreso.

Lo observado en el mapa se puede traducir a un indicador de autocorrelación espacial. ${ }^{3}$ En particular, es posible estimar el indicador de Moran (1948), un estadístico que mide qué tan dispersos o agrupados se encuentran los valores similares de un atributo específico. ${ }^{4}$ En este caso, se estima si existen clusters de participación laboral femenina, esto es, si zonas de alta participación están rodeadas de zonas de alta participación, y al contrario, si zonas de baja participación están rodeadas de baja participación laboral femenina.

Figura 2. AUTOCORRELACIÓN ESPACIAL DE LAS TASAS DE PARTICIPACIÓN LABORAL FEMENINA. REGIÓN METROPOLITANA, AÑO 2017

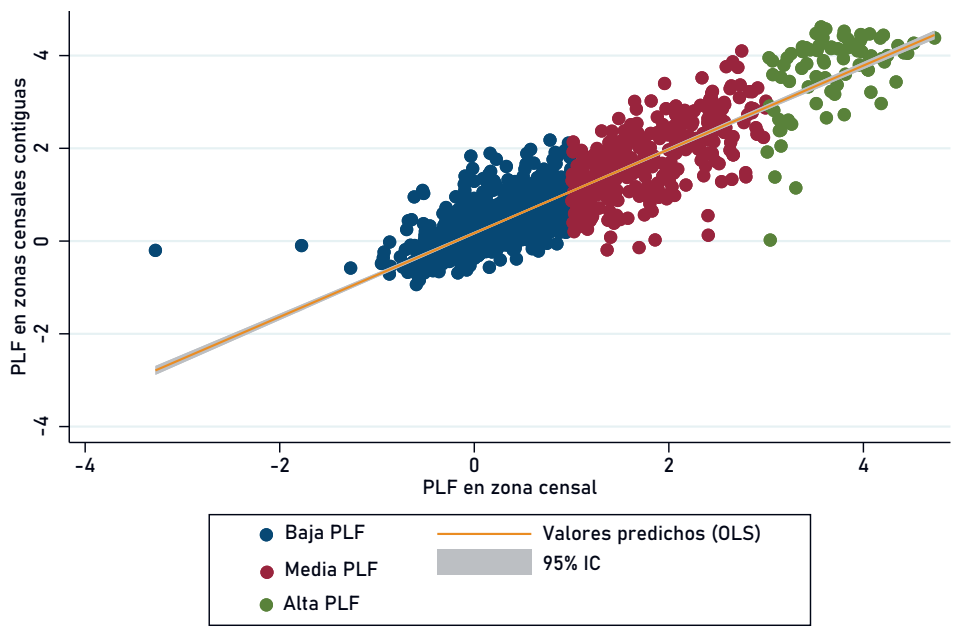

PLF: Participación Laboral Femenina (estandarizada) Índice de Moran I: 0,89

Fuente: Elaboración propia en base a Censo 2017, INE.

\footnotetext{
${ }^{3}$ El Anexo 1 presenta la autocorrelación espacial de la tasa de participación laboral femenina, desagregado por región. En todas las regiones se observa una autocorrelación positiva, aunque esta es menor en zonas de menor densidad poblacional, como ocurre en las regiones de Atacama, Aysén y Magallanes.

${ }^{4} \mathrm{El}$ indicador puede tomar valores entre -1 y 1 , donde -1 indica dispersión perfecta del atributo, 0 sugiere distribución aleatoria del atributo y 1 indica una agrupación perfecta de valores similares del atributo.
} 
Para obtener el indicador de Moran, primero se establece el vecindario de cada zona censal. En este caso, se definen como zonas censales vecinas aquellas zonas censales que son adyacentes, es decir, comparten parte de sus perímetros. En segundo lugar, se estima la correlación existente entre la tasa de participación laboral femenina de cada zona censal y la tasa de participación laboral femenina exhibida en las zonas censales vecinas. ${ }^{5}$ La representación gráfica de esta relación se presenta en la Figura 2, en la cual se aprecia una clara correlación positiva entre la tasa de participación propia de cada zona censal (representada en el eje $\mathrm{x}$ ) y la tasa de participación de las zonas censales vecinas (representadas en el eje y).

Figura 3. NIVEL DE PARTICIPACIÓN LABORAL FEMENINA (PLF) Y CORRELACIÓN AUTOESPACIAL EN ZONAS CENSALES. REGIÓN METROPOLITANA, AÑO 2017

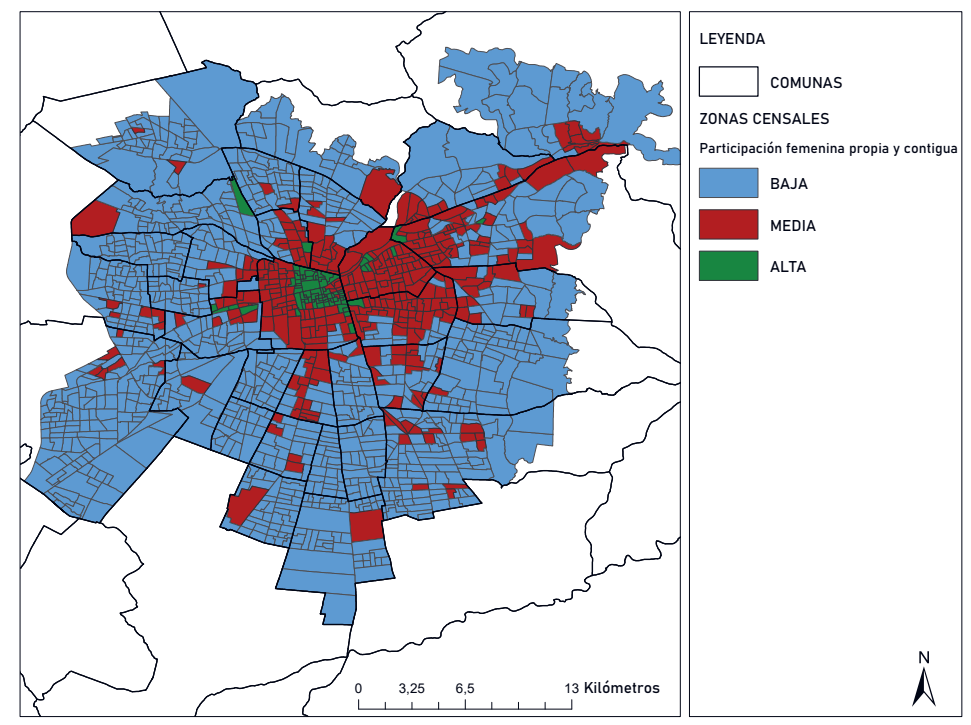

Fuente: Elaboración propia en base a Censo 2017, INE.

El indicador de Moran estimado es igual a 0,89 y sugiere que las tasas de participación laboral femenina se encuentran, efectivamente, agrupadas según valores similares. ${ }^{6}$ Además, la Figura 2 distingue a

\footnotetext{
${ }^{5}$ Esta correlación se estima sobre los valores estandarizados, esto es, una vez que se resta la media y divide por su desviación estándar.

${ }^{6}$ El indicador es estadísticamente significativo. Los resultados de la simulación utilizada para probar su significancia se presentan en el Anexo 2.
} 
aquellas zonas censales que tienen una alta, media o baja tasa de participación laboral femenina en colores verde, rojo y azul, respectivamente. Esta clasificación permite ilustrar en el mapa dónde se encuentran estas zonas censales. Se observa que aquellas zonas de alta participación laboral femenina que están rodeadas de alta participación se encuentran principalmente en el centro cívico de la ciudad (véase Figura 3).

El fenómeno anterior no ocurre en el caso de los hombres, quienes exhiben altas tasas de participación laboral de manera transversal en el territorio. En efecto, en la Figura 4 se presentan las tasas de participación laboral masculina según zona censal en la Región Metropolitana, utilizando las categorías presentadas para las mujeres en la Figura 1. En este caso, no es posible observar clusters de participación laboral como en el caso de las mujeres.

Figura 4. TASA DE PARTICIPACIÓN LABORAL MASCULINA SEGÚN ZONAS CENSALES. REGIÓN METROPOLITANA, AÑO 2017

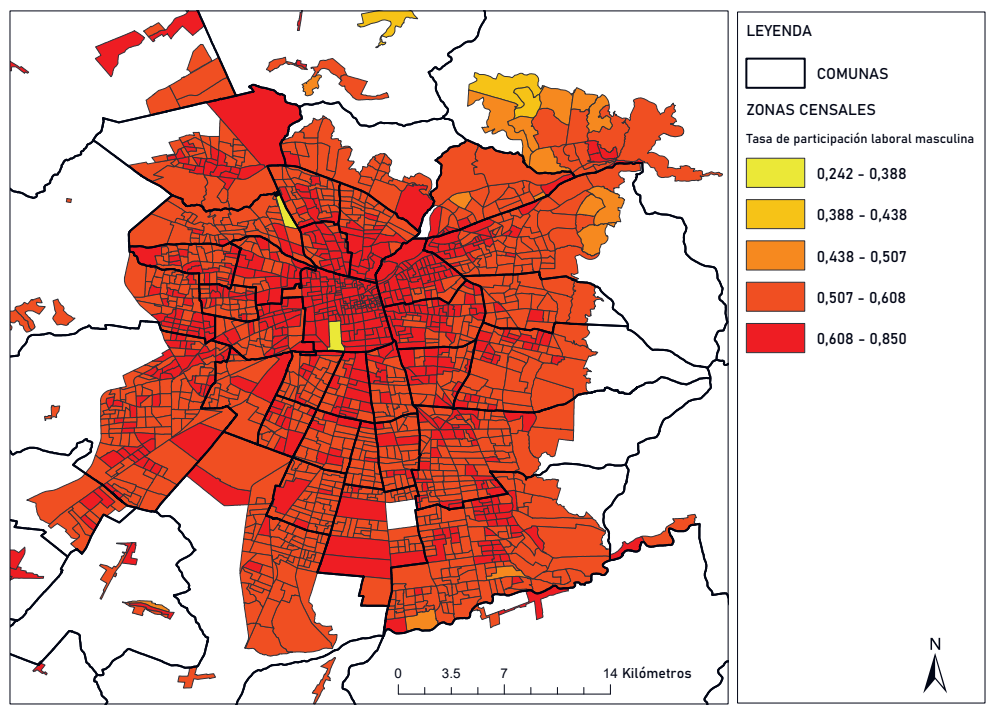

Fuente: Elaboración propia en base a Censo 2017, INE.

Estos análisis evidencian que la distribución de las tasas de participación laboral femenina, están efectivamente agrupadas en grupos de valores similares. Asimismo, se constata que este fenómeno es particular 
para el caso de las mujeres y no ocurre en el caso de los hombres. Se justifica, así, la relevancia de un enfoque territorial a nivel de zonas censales para analizar interacciones sociales entre mujeres.

\section{Interacciones sociales dentro del barrio y comportamiento individual}

En cada barrio se establecen relaciones sociales significativas y es el lugar donde se constituyen las comunidades locales (Forrest 2008). En efecto, los vecindarios constituyen una forma natural de interacción social y permiten ubicar social y espacialmente a las mujeres dentro de una red social específica. 7 Más aún, existe evidencia que sugiere que sujetos cercanos geográficamente están más relacionados que sujetos distantes, como ya lo indicaba Tobler (1976) en la primera ley de la geografía. Esta premisa ha sido utilizada ampliamente en econometría espacial para modelar la dependencia observada entre observaciones cercanas (LeSage 2008). Además, Patacchini, Picard y Zenou (2015) presentan evidencia sobre la importancia de la proximidad para las interacciones sociales, indicando que la intensidad de los contactos depende de la ubicación. Los autores demuestran que se tiende a interactuar más con los individuos que están geográficamente más cerca, aun en presencia de interacciones digitales (Goldenberg y Levy 2009). Estudios anteriores de Wellman (1996, 2001) presentan resultados similares.

Es de notar que las interacciones sociales se definen como la interdependencia directa entre las decisiones de los individuos, la cual no está mediada por los mercados o contratos (Brock y Durlauf 2001b). La influencia de las interacciones sociales sobre el comportamiento individual puede explicarse por tres factores principales. En primer lugar, la existencia de factores psicológicos, como la inspiración o la identidad. Una mujer se sentiría inducida a trabajar como aquellas mujeres en quienes se ve representada o hacia quienes siente admiración (Akerlof y Kranton 2000). En segundo lugar, la existencia de interdependencias en las restricciones que enfrentan las mujeres puede afectar los costos de elegir participar en el mercado laboral cuando otras mujeres están trabajando (Manski 2000).

\footnotetext{
${ }^{7}$ Naturalmente, cada persona posee distintas redes sociales relevantes, como la familia o amigos, no necesariamente vecinos. No obstante, este estudio se enfoca en las redes sociales de vecinas.
} 
Y tercero, existe un mecanismo de transmisión de información que puede afectar las percepciones que tienen las mujeres sobre las oportunidades en el mercado laboral y las ventajas del trabajar (Durlauf 2004).

Muy pocos estudios empíricos han estudiado el rol de las interacciones sociales a nivel de vecindario en el contexto del mercado laboral para las mujeres. ${ }^{8}$ Más aún, la evidencia existente considera solo países desarrollados. En efecto, Maurin y Moschion (2009), con datos para Francia, estiman la elasticidad de la oferta de trabajo femenina frente a cambios en la participación laboral de vecinas, utilizando como instrumento la composición de género de los hijos e hijas de las vecinas. Sus resultados indican que un aumento de 10 puntos porcentuales en la participación de las vecinas aumenta la probabilidad propia de participar en el mercado laboral de 6 puntos porcentuales.

Luego, Mota et al. (2016) establecen diferentes definiciones de pares en función de la edad, el género, la presencia y la edad de los hijos, el estado civil y el nivel educativo, y utilizan una variación temporal en la situación laboral individual de los pares y no pares para la identificación. Los resultados indican efectos sociales positivos: una trabajadora adicional en el barrio aumenta la probabilidad individual de una mujer de participar en el mercado laboral en 4,5 puntos porcentuales.

Finalmente, Nicoletti et al. (2018) estiman el efecto de las redes en el comportamiento de madres en el mercado laboral. Con datos administrativos noruegos que incluyen información sobre los vecinos y sus relaciones familiares, los autores asumen que una madre interactúa con sus vecinos y con su familia, pero no con la familia del vecino. Los resultados indican que solo primos y hermanas tienen efectos significativos sobre el número de horas trabajadas por madres con hijos en edad preescolar.

Este artículo contribuye a esta rama de la literatura sobre interacciones sociales, aportando evidencia cuantitativa sobre la relación que existe entre la participación de las vecinas y la decisión individual de una mujer de integrarse al mercado del trabajo, en una economía emergente y en un contexto de tasas de participación laboral femenina particularmente bajas.

\footnotetext{
${ }^{8}$ Estudios previos se han enfocado en cuantificar el efecto de las características de las redes sociales sobre resultados en el mercado laboral, tales como la obtención de empleo o nivel de salarios a través de redes informales (Boucher y Goussé 2019; Contreras et al. 2019; Topa 2011), así como también las decisiones ocupacionales, horas de trabajo e ingresos de hombres, principalmente (Bayer, Ross y Topa 2008; Conley y Topa 2007; Guerra y Mohnen 2014; Oreopoulos 2003; Rosenbaum et al. 1999; Schmutte 2015; Topa 2001; Vandecasteele y Fasang 2020; Weinberg, Reagan y Yankow 2004).
} 


\section{Tipos de lazos sociales que se conforman en el barrio}

Al estudiar interacciones sociales, no solo es importante detectar si estas ocurren, sino que también es relevante analizar cuál es el tipo de lazo social que se crea entre quienes interactúan. Siguiendo a Granovetter (1973) y su trabajo sobre la importancia de los lazos débiles, se extrae que la fortaleza de un lazo entre individuos depende de cuatro dimensiones: tiempo que comparten, intensidad emocional del vínculo, intimidad y confianza mutua, y reciprocidad de la relación. Entonces, resulta relevante analizar las interacciones sociales según la fortaleza del lazo.

Según su teoría y posterior evidencia empírica (Granovetter 1973, 1983, 1995; Marsden y Campbell 1984), los lazos débiles, con quienes se tiene muy poco contacto y escaso vínculo emocional, serían relevantes para generar puentes que dan acceso a información nueva y diferente de aquella provista por los lazos más fuertes, como la familia o amigos cercanos. En el contexto del mercado laboral, se ha evidenciado que la búsqueda de trabajo sería más efectiva en presencia de interacciones sociales entre personas con lazos más débiles (Brown y Konrad 2001; Greenberg y Fernández 2016; Montgomery 1992).

Esta investigación se basa en la definición de Granovetter (1973). Junto con los datos de ELSOC (COES 2017) se construye un indicador que da cuenta del tipo de lazo social que tienen las mujeres encuestadas con las personas que residen en su barrio. Específicamente, este indicador resulta de una combinación lineal de las cuatro dimensiones señaladas, asignando igual peso a cada una de ellas. ${ }^{9}$

El indicador resultante toma valores entre 0 y 1, donde 1 es máxima fortaleza y 0 máxima debilidad del lazo social conformado entre cada mujer y sus vecinos. La mediana de este indicador es 0,62. La Figura 5 presenta el promedio de cada dimensión del indicador y la línea punteada muestra la mediana para la muestra bajo estudio.

\footnotetext{
${ }^{9}$ La dimensión (i) cantidad de tiempo hace referencia a la frecuencia de contacto entre la mujer con sus vecinos. La dimensión (ii) intensidad emocional alude a la cercanía que tienen dos personas. La dimensión (iii) intimidad se refiere a la variedad de temas tratados y confianza para hablar de temas personales. La dimensión (iv) reciprocidad consiste en la existencia de servicios mutuos y la correspondencia de la relación. El Anexo 3 presenta en detalle la metodología de construcción del indicador de fortaleza de lazos sociales.
} 
Figura 5. DIMENSIONES QUE COMPONEN EL INDICADOR DE FORTALEZA DE LAZOS SOCIALES Y SU MEDIANA, AÑO 2017

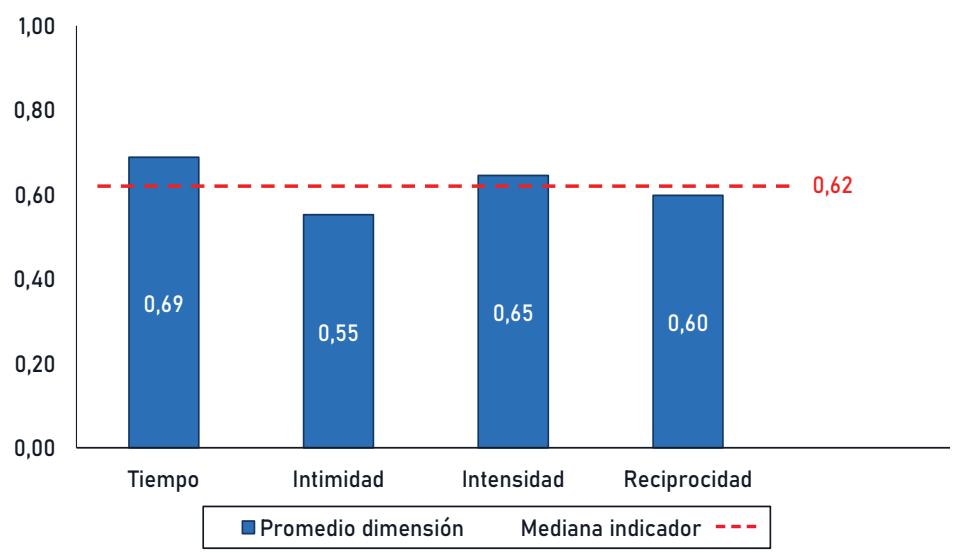

Fuente: Elaboración propia en base a ELSOC (COES 2017).

La construcción de este tipo de indicadores contribuye a la literatura sobre cohesión en el contexto barrial (Casakin, Hernández y Ruiz 2015; Méndez y Otero 2018; Méndez et al. 2020), la cual indica la calidad de la convivencia y la disposición de las personas para enfrentar distintos aspectos de la vida en común (Forrest y Kearns 2001). Esto es relevante ya que, a mayor cohesión, se debería observar un incremento en las interacciones sociales dentro de un barrio, demostrando la existencia de un capital social específico, que permite alcanzar objetivos individuales y colectivos de manera colaborativa (Ritzen, Easterly y Woolcock 2000; Novy, Swiatek y Moulaert 2012).

\section{Modelo y aplicación empírica}

\section{Elección binaria con interacciones sociales}

El modelo teórico consiste en un modelo estándar de elección binaria individual (participar o no en el mercado laboral), al que se incluyen las interacciones sociales (Brock y Durlauf 2001a). Específicamente, las interacciones sociales entran al modelo en la función de utilidad individual, a través de las elecciones de todas las mujeres del barrio. 
Formalmente, considere una población de / mujeres indexadas por $i$ $=1,2, \ldots, l$, dentro de cada barrio indexado por $g, g=1,2, \ldots . G$. Cada mujer $i$ debe elegir una acción binaria, denotada por $y_{i g}$, en un tiempo común a las otras mujeres. Entonces, se puede escribir $Y_{i g}=0,1$, el set de estrategias de la mujer $i$, donde 1 corresponde a participar en el mercado laboral, 0 si decide no hacerlo. El set de acciones de las vecinas, sin incluir a la mujer $i$, está denotado por $y_{-i g}=y_{1 g}, y_{2 g}, \ldots, y_{i-1}, y_{i+1 g}, \ldots, y_{l g}$.

Cada mujer $i$ escoge $y_{i g}$ con el objetivo de maximizar su utilidad individual, la cual depende de las características individuales observables (vector $x_{i g}$ ), de heterogeneidad no observable que depende de la decisión individual, $\epsilon_{i g}\left(y_{i g}\right)$, de la decisión individual $y_{i g}$, y de las decisiones de las otras mujeres en el barrio $\mathrm{y}_{-i g}$ :

$$
V\left(y_{i g}, x_{i g}, y_{-i g}, \epsilon\left(y_{i g}\right)\right)=u\left(y_{i g}, x_{i g}\right)+s\left(y_{i g}, y_{-i g}\right)+\epsilon_{i g}\left(y_{i g}\right)
$$

La ecuación (E1) asume que la utilidad individual es lineal en tres elementos: la utilidad individual, $u\left(y_{i g}, x_{i g}\right)$, la utilidad social, $s\left(y_{i g}, y_{-i g}\right), y$ la heterogeneidad no observable de cada mujer, $\epsilon_{i g}\left(y_{i g}\right)$.

Adicionalmente, se puede definir el componente de utilidad social de la siguiente manera:

$$
s\left(y_{i g}, y_{-i g}\right)=y_{i g}\left[\frac{\gamma}{(I-1)} \sum_{j \neq i} y_{j g}+d_{g}\right]
$$

Esta forma funcional es una interacción multiplicativa entre la elección individual $y_{-i g}$, el promedio de las decisiones de las vecinas, $\bar{y}_{-i g=} \frac{1}{(I-1)} \sum_{j \neq i} y_{j g}$ y un efecto fijo del barrio, $d_{g}$. Según Brock y Durlauf (2001a), esta especificación representa un caso de spillovers proporcionales, donde el cambio porcentual en la utilidad de una mujer $i$, dado un cambio en el nivel medio de las decisiones de las vecinas, es constante:

$$
\frac{\partial^{2} s\left(y_{i g}, y_{-i g}\right)}{\partial y_{i g} \partial \bar{y}_{-i g}}=\gamma
$$

Notar que si $\gamma>0$, entonces existe una complementariedad estratégica entre las opciones de cualquier par de mujeres. En este caso, la utili- 
dad de participar $\left(y_{i g}=1\right)$ cuando cualquier otra mujer $j$ participa $\left(y_{j g}=1\right)$ es mayor que la utilidad de participar cuando cualquier otra mujer $j$ no participa $\left(y_{j g}=0\right) .{ }^{10}$ Por el contrario, si $\gamma<0$, las elecciones de cualquier par de mujeres son sustitutas estratégicas. Luego, si $\gamma=0$, no hay efectos sociales sobre la elección de la mujer $i$.

Este modelo asume que las mujeres no coordinan sus formas de actuar. De hecho, cada mujer toma decisiones para maximizar su propia utilidad dada una expectativa del promedio de las elecciones de su grupo de referencia (el barrio), la cual es independiente de $\epsilon_{i g}\left(y_{i g}\right)$, para todo $i$ del grupo $g$. Además, se asume que el término de utilidad individual es una función lineal de las características exógenas $x_{i g}$. Por lo tanto, condicional a la elección individual de la mujer $i$, los términos de utilidad individual específica son: $u\left(1, x_{i g}\right)=\beta_{1} x_{i g}$ y $u\left(0, x_{i g}\right)=\beta_{0} x_{i g}$.

Por consiguiente, la mejor respuesta de la mujer $i$, condicional a las decisiones de todas las otras mujeres, se obtiene a partir de la diferencia entre la utilidad de escoger $y_{i g}=1$ y la utilidad de escoger $y_{i g}=0$ :

$$
y_{i g}^{*}=V\left(1, x_{i g}, y_{-i g}, \epsilon_{i g}(1)\right)-V\left(0, x_{i g}, y_{-i g}, \epsilon_{i g}(0)\right)
$$

Reescribiendo la ecuación (E4):

$$
\begin{aligned}
y_{i g}^{*} & =u\left(1, x_{i g}\right)+s\left(1, y_{-i g}\right)+\epsilon_{i g}(1)-u\left(0, x_{i g}\right)-s\left(0, y_{-i g}\right)-\epsilon_{i g}(0) \\
& =\beta_{1} x_{i g}+\frac{\gamma}{I-1} \sum_{j \neq i} y_{j g}+d_{g}+\epsilon_{i g}(1)-\beta_{0} x_{i g}-\epsilon_{i g}(0) \\
& =\beta x_{i g}+\gamma \bar{y}_{-i g}+d_{g}+\epsilon_{i g}
\end{aligned}
$$

Donde $\beta=\beta_{1}-\beta_{0}$ y $\epsilon_{i g}=\epsilon_{i g}(1)-\epsilon_{i g}(0)$. Por lo tanto, la mejor respuesta de la mujer $i$ se puede representar de la siguiente manera:

$$
y_{i g}= \begin{cases}1, & \text { si } y_{i g}^{*}>0 \\ 0, & \text { si } y_{i g}^{*} \leq 0\end{cases}
$$

Se puede demostrar que el perfil de estrategias $y_{i g}$ es un equilibrio de Nash de estrategias puras, si y solo si, este es consistente con la ecuación (E6) para toda mujer $i$.

\footnotetext{
${ }^{10}$ Ver demostración en Anexo 4.
} 


\section{Especificación econométrica}

Como se ha señalado, cada mujer i vive en un barrio $g=1,2, \ldots, G$ y se asume que las interacciones sociales ocurren dentro de los límites del barrio, el cual corresponde a la zona censal de residencia. Desde luego, para la mujer $i$ existen otras redes sociales relevantes. No obstante, se asume que las interacciones sociales entre vecinas son importantes para cada mujer y que estas ocurren a la escala de barrio. Es decir, las vecinas más cercanas son más importantes que las vecinas lejanas, residentes en zonas censales adyacentes o más distantes.

Estos supuestos han sido utilizados previamente para construir redes sociales (Aizer y Currie 2004; Bayer et al. 2008; Guerra y Mohnen 2014; Schmutte 2015), basándose en la premisa de que las interacciones importantes ocurren dentro de ubicaciones geográficas pequeñas (Topa 2011). ${ }^{11}$ Si bien existen redes cercanas que no son locales, el contacto frecuente con los vecinos sitúa la proximidad como un factor relevante para estudiar las interacciones sociales (Wellman 1996, 2001).

Formalizando, a partir de las ecuaciones (E5) y (E6) del modelo teórico, se puede estimar la función de mejor respuesta, especificando el modelo empírico de la ecuación (E7), donde la variable $y_{\text {ig }}$ toma el valor 1 si la mujer $i$ que vive en el vecindario $g$ participa en la fuerza laboral y 0 si no lo hace:

$$
\begin{aligned}
y_{i g} & =1\left\{y_{i g}^{*}>0\right\} \\
& =1\left\{\beta x_{i g}+\gamma \bar{y}_{-i g}+d_{g}+\epsilon_{i g}>0\right\}
\end{aligned}
$$

Este modelo de participación en la fuerza laboral es similar al modelo de Blundell y Powell (2004), donde el indicador binario, $y_{\text {ig, en la }}$ ecuación (E7) es una función de diferentes variables demográficas observables, determinantes del salario como el nivel de educación, y un proxy de los ingresos no laborales. No obstante, además en este caso se incluye como factor explicativo la tasa de participación laboral de todas las mujeres del barrio de residencia, sin considerar a la mujer $i$.

Entonces, como la variable dependiente del modelo es una variable binaria, el objetivo es estimar la siguiente probabilidad:

\footnotetext{
${ }^{11}$ En Estados Unidos existe evidencia que indica que los individuos cuentan con una alta proporción de amigos cercanos, parientes o compañeros de trabajo en el barrio de residencia (Guest y Lee 1983; Lee y Campbell 1999).
} 


$$
P\left(y_{i g}=1 \mid x_{i g}, \bar{y}_{-i g}, d_{g}\right)=P\left(\beta x_{i g}+\gamma \bar{y}_{-i g}+d_{g}>\epsilon_{i g}\right)
$$

La ecuación (E8) indica que la probabilidad de participar en el mercado laboral es una función de un vector de características observables de la mujer $i, x_{i g}$ la tasa de participación laboral de las vecinas que viven en su barrio, $\bar{y}_{-i g}$, y características no observables del barrio, ${ }^{12} d_{g}$, y un término de error que representa la heterogeneidad no observable, $\epsilon_{i g}$.

El vector $x_{i g}$ incluye edad, nivel de educación, si tiene pareja, presencia de niños menores de 6 años en el hogar, si es la principal sostenedora del hogar, ingreso no laboral per cápita del hogar. También se incluyen tres variables culturales, que podrían estar asociadas a su inserción en el mercado del trabajo. Estas indican su grado de acuerdo a tres afirmaciones: (i) Una madre que trabaja puede establecer una relación tan cálida y sólida con sus hijos como una madre que no trabaja; (ii) Los hombres deberían asumir un mayor grado de responsabilidad en el trabajo doméstico que el que asumen actualmente; y (iii) Los hombres deberían asumir un mayor grado de responsabilidad en el cuidado de los niños que el que asumen actualmente.

Este modelo se estima para todas las mujeres que tienen hasta educación secundaria completa. ${ }^{13}$ Con el fin de estudiar la heterogeneidad de los efectos de las interacciones sociales sobre las decisiones individuales, el modelo se estima de manera separada para aquellas mujeres que tienen un indicador de lazos sociales en el barrio sobre la mediana y bajo la mediana. Esto, para analizar el rol que tiene la fortaleza de los lazos entre vecinos sobre la decisión individual de participar en el mercado del trabajo.

\footnotetext{
12 En la práctica, el tamaño muestral no permite realizar estimaciones con efectos fijos a nivel de vecindario, por lo que se incluye un indicador binario que toma el valor 1 si la mujer reside en la Región Metropolitana y 0 si no lo hace. Además, se incluye una variable con la proporción de mujeres en edad de trabajar que viven en el barrio, para controlar por la heterogeneidad del tamaño de las zonas censales y potenciales excesos de oferta laboral femenina a nivel local.

${ }^{13}$ Mujeres más educadas y hombres presentan altas tasas de participación laboral. Su decisión de integrarse al mercado del trabajo parece estar relacionada con otros factores, lo que hace irrelevante la participación laboral promedio de vecinas o vecinos residentes en su barrio. El Anexo 5 presenta los resultados de la estimación de los factores asociados a la probabilidad de participar en el mercado laboral, según género y nivel educativo. Los resultados indican que la tasa de participación laboral de mujeres u hombres del barrio solo es relevante para mujeres que tienen hasta educación secundaria completa. Esto es, el coeficiente estimado no es estadísticamente significativo para hombres, independientemente de su nivel educativo, ni para mujeres con educación terciaria o más.
} 
El parámetro de interés es $\gamma$, el cual representa el grado de complementariedad o de sustitución estratégica entre la elección de la mujer $i$ y las elecciones de las vecinas que residen en la misma zona censal. Según la literatura sobre efectos de pares, este parámetro capturaría el efecto social endógeno (Manski 1993), el cual representa la influencia de las decisiones de las vecinas sobre la decisión individual de trabajar.

Entonces, se estima por máxima verosimilitud un modelo Probit. Es decir, en la ecuación (E9) se asume que la probabilidad de participar en el mercado laboral es una función no lineal en los parámetros a estimar. Específicamente, se asume una función de distribución acumulada normal, $\Phi$, la cual asegura que las predicciones del modelo se encuentran entre 0 y 1 , fundamental al tratarse de la estimación de una probabilidad.

$$
P\left(y_{i g}=1 \mid x_{i g}, \bar{y}_{-i g}, d_{g}\right)=\Phi\left(\beta x_{i g}+\gamma \bar{y}_{-i g}+d_{g}+\epsilon_{i g}\right)
$$

Se debe señalar que la variable $\bar{y}_{-i g}$ en la ecuación (E9) es una variable potencialmente endógena. En particular, existiría un problema de simultaneidad, ya que la decisión de la mujer $i$ afecta las decisiones de las vecinas ' $\bar{y}_{-i g}$, y viceversa (efecto reflejo). También, la existencia de efectos correlacionados y la clasificación no aleatoria de las mujeres en el barrio, hacen que la estimación directa de $\gamma$ no represente exclusivamente un efecto causal (Manski 1993).

Por lo tanto, la interpretación del parámetro estimado de $\gamma$ debe hacerse con cautela, y considerarse como una correlación entre la decisión individual de participación laboral y la decisión grupal de las vecinas residentes en una misma zona censal. La riqueza de los datos que asocian las características de la zona censal exacta de cada mujer, permite determinar si existe esta correlación y establecer bases que orienten investigaciones futuras con foco en identificar el efecto causal.

\section{Datos y estadísticas descriptivas}

La investigación que expone este artículo utiliza datos provenientes de dos fuentes que se vinculan entre sí para contar con rica información demográfica de mujeres y con información territorial a nivel de zonas censales.

En primer lugar, se utilizan los datos de ELSOC (COES 2016, 2017), una encuesta panel y representativa de la población nacional urbana, 
que está diseñada para analizar la evolución del conflicto y cohesión en la sociedad chilena. La encuesta contiene siete módulos temáticos: territorio, redes y actitudes sociales, ciudadanía y democracia, desigualdad y legitimidad, conflicto social, salud y bienestar, y caracterización sociodemográfica.

Para la redacción de este artículo se utiliza la segunda ronda de ELSOC del año 2017, la cual coincide con el levantamiento del Censo 2017 del Instituto Nacional de Estadísticas (INE). De esta manera, es posible vincular características de la zona censal de residencia a cada encuestado en ELSOC.

Específicamente, con la información a nivel de personas del Censo 2017, es posible extraer el total de mayores de 15 años de edad que participan en el mercado laboral a nivel de zonas censales, por tramos de edad y género. Luego, con la cartografía digital urbana del Censo 2017 del INE, se utilizan herramientas de procesamiento de información geográfica para construir los mapas de participación laboral femenina y masculina por zona censal. En los datos, cada zona censal tiene asociada su información sobre mujeres y hombres en la fuerza de trabajo, y total de mujeres y hombres que residen en ella. Esta información se vincula a cada observación en ELSOC en 2017.14

Con la información vinculada de ELSOC y Censo 2017 para cada mujer en la muestra, se construye la tasa de participación a nivel de su zona censal de residencia. Tal como se señaló en la sección metodológica, la tasa de participación laboral femenina en la zona censal de la mujer $i$, considera a todas las mujeres $j$, con $j \neq i$. De manera similar, se calcula la participación laboral masculina a nivel de zona censal para cada hombre en la muestra de ELSOC, para realizar el ejercicio de selección de la muestra bajo análisis, presentado en el Anexo $5 .^{15}$

\footnotetext{
${ }^{14}$ Se agradece al Equipo Técnico de ELSOC de COES, encargado de realizar la vinculación de estos datos, bajo estrictos protocolos de seguridad que garantizan la anonimidad de los datos aquí utilizados.

${ }^{15}$ La segunda ronda de ELSOC del año 2017 contiene 2.473 observaciones, 951 hombres y 1.522 mujeres. Algunas observaciones no cuentan con toda la información necesaria para este estudio, por lo que no se consideran 286 observaciones. Entonces, los análisis se realizan a partir de una muestra de 845 hombres y 1.342 mujeres, en total, 2.187 observaciones. Todos los análisis, estadísticas descriptivas y regresiones econométricas se realizan utilizando el ponderador muestral de la segunda ronda, que ajusta en base a la probabilidad de selección, no respuesta y población objetivo estimada a nivel regional y según sexo.
} 
Tabla 1. MEDIA Y (DESVIACIÓN ESTÁNDAR) DE VARIABLES DE INTERÉS SEGÚN NIVEL EDUCACIONAL

\begin{tabular}{|c|c|c|c|c|}
\hline \multirow[t]{2}{*}{ Variable } & \multirow{2}{*}{$\begin{array}{l}\text { Todas las } \\
\text { mujeres }\end{array}$} & \multicolumn{2}{|c|}{ Nivel educativo } & \multirow{2}{*}{ Diferencia } \\
\hline & & $\begin{array}{c}\text { Educación } \\
\text { terciaria }\end{array}$ & $\begin{array}{l}\text { Educación } \\
\text { secundaria }\end{array}$ & \\
\hline & (1) & (2) & (3) & (3)-(2) \\
\hline \multirow[t]{2}{*}{ 1: Participa en mercado laboral 2017} & 0,59 & 0,71 & 0,52 & $-0,19 * * *$ \\
\hline & $(0,49)$ & $(0,46)$ & $(0,50)$ & $(0,04)$ \\
\hline \multirow[t]{2}{*}{ Edad } & 46,52 & 41,46 & 49,79 & $8,32^{* * *}$ \\
\hline & $(14,80)$ & $(14,63)$ & $(13,98)$ & $(1,20)$ \\
\hline \multirow[t]{2}{*}{ 1:Tiene pareja } & 0,51 & 0,41 & 0,58 & $0,17^{* * *}$ \\
\hline & $(0,50)$ & $(0,49)$ & $(0,49)$ & $(0,04)$ \\
\hline \multirow[t]{2}{*}{ Número de niños < 6 años } & 0,30 & 0,28 & 0,32 & 0,04 \\
\hline & $(0,64)$ & $(0,64)$ & $(0,64)$ & $(0,07)$ \\
\hline \multirow[t]{2}{*}{ 1: Principal sostenedor del hogar } & 0,41 & 0,44 & 0,39 & $-0,05$ \\
\hline & $(0,49)$ & $(0,50)$ & $(0,49)$ & $(0,04)$ \\
\hline \multirow[t]{2}{*}{ Log ingreso no laboral per cápita } & 7,13 & 6,50 & 7,54 & 1,04 \\
\hline & $(10,48)$ & $(11,41)$ & $(9,82)$ & $(0,97)$ \\
\hline \multirow[t]{2}{*}{ 1: Reside en RM } & 0,44 & 0,50 & 0,40 & $-0,09 * *$ \\
\hline & $(0,50)$ & $(0,50)$ & $(0,49)$ & $(0,04)$ \\
\hline \multirow[t]{2}{*}{ Calidez madre trabajadora con hijos ${ }^{\mathrm{a}}$} & 0,57 & 0,59 & 0,56 & $-0,03$ \\
\hline & $(0,49)$ & $(0,49)$ & $(0,50)$ & $(0,05)$ \\
\hline \multirow[t]{2}{*}{ Hombres y responsabilidades domésticas ${ }^{b}$} & 0,95 & 0,96 & 0,94 & $-0,02$ \\
\hline & $(0,22)$ & $(0,20)$ & $(0,23)$ & $(0,01)$ \\
\hline \multirow[t]{2}{*}{ Hombres y cuidado infantilc } & 0,96 & 0,97 & 0,96 & $-0,01$ \\
\hline & $(0,19)$ & $(0,17)$ & $(0,21)$ & $(0,01)$ \\
\hline \multirow[t]{2}{*}{ Tasa PLF zona censal 2017} & 0,40 & 0,42 & 0,39 & $-0,03 * * *$ \\
\hline & $(0,06)$ & $(0,07)$ & $(0,05)$ & $(0,01)$ \\
\hline \multirow[t]{2}{*}{ Proporción de mujeres en zona censal 2017} & 0,52 & 0,52 & 0,52 & $-0,00 * *$ \\
\hline & $(0,02)$ & $(0,02)$ & $(0,01)$ & $(0,00)$ \\
\hline \multirow[t]{2}{*}{ 1: Visita a sus vecinos } & 0,68 & 0,68 & 0,69 & 0,00 \\
\hline & $(0,46)$ & $(0,47)$ & $(0,46)$ & $(0,04)$ \\
\hline \multirow[t]{2}{*}{ 1: Se identifica con sus vecinos } & 0,63 & 0,57 & 0,67 & $0,10^{* *}$ \\
\hline & $(0,48)$ & $(0,50)$ & $(0,47)$ & $(0,04)$ \\
\hline \multirow[t]{2}{*}{ Fortaleza de lazos sociales con vecinos } & 0,61 & 0,61 & 0,61 & 0,00 \\
\hline & $(0,25)$ & $(0,25)$ & $(0,25)$ & $(0,02)$ \\
\hline \multirow[t]{2}{*}{ 1: Educación secundaria completa o menos } & 0,61 & & & \\
\hline & $(0,49)$ & & & \\
\hline Observaciones & 1,342 & 410 & 932 & 1,342 \\
\hline
\end{tabular}

Notas: a) De acuerdo con la afirmación: 'Una madre que trabaja puede establecer una relación tan cálida y sólida con sus hijos como una madre que no trabaja'; b) De acuerdo con la afirmación: 'Los hombres deberían asumir un mayor grado de responsabilidad en el trabajo doméstico que el que asumen actualmente', y c) De acuerdo con la afirmación: 'Los hombres deberían asumir un mayor grado de responsabilidad en el cuidado de los niños que el que asumen actualmente'. Se reportan los $p$-values del test $t$ $\left({ }^{* * *} p<0,01 ;{ }^{* *} p<0,05 ;{ }^{*} p<0,1\right)$.

Fuente: Elaboración propia en base a ELSOC (COES 2016, 2017). 
Como se ha señalado, la muestra de mujeres de ELSOC 2017 que se considera en la aplicación empírica corresponde a todas aquellas mujeres que tienen hasta educación secundaria completa. Las mujeres que tienen algo de educación terciaria o más, no se incluyen en la muestra, ya que ellas presentan altas tasas de participación laboral y tienen características observables diferentes a las mujeres con menores niveles de educación (véase Tabla 1).

La Tabla 1 indica que las mujeres que tienen hasta educación secundaria completa presentan una tasa de participación laboral femenina que es hasta 19 puntos porcentuales menos que las mujeres que tienen educación terciaria o más (52\% frente a $71 \%$ ). Asimismo, se extrae que las mujeres que tienen hasta educación secundaria completa tienen en promedio 8 años más (49 frente a 41 años de edad), una mayor proporción tiene pareja ( $58 \%$ frente a $41 \%$ ), una proporción mayor vive fuera de la Región Metropolitana (50\% frente a 40\%), se encuentran rodeadas de mujeres con tasas de participación laboral menores (39\% frente a 42\%) y se identifican en mayor medida con sus vecinas ( $67 \%$ frente a $57 \%$ ), que aquellas mujeres con mayores niveles de escolaridad.

No se encuentran diferencias estadísticamente significativas en la presencia de niños menores de 6 años, en la proporción que es la principal sostenedora del hogar, ingresos no laborales del hogar per cápita, y tampoco en cuanto a las variables culturales relacionadas con su percepción de la relación de una madre trabajadora y sus hijos, o en relación al rol del hombre respecto del trabajo doméstico y el cuidado infantil.

\section{Resultados}

Los resultados que se presentan a continuación consideran la muestra de mujeres que tienen hasta educación secundaria completa. Para analizar la heterogeneidad de los resultados según fortaleza de lazo social conformado con los vecinos, las estimaciones se realizan por separado para aquellas mujeres que tienen un indicador de lazo social con vecinos menor a la mediana de 0,62 y para aquellas que tienen un indicador de lazo social mayor o igual a la media.

La Tabla 2 expone los resultados de las estimaciones. En particular, se reportan los efectos marginales del modelo Probit. Las columnas (1) y (2) presentan los resultados para mujeres con lazos débiles en el barrio, 
Tabla 2. ESTIMACIÓN DE LA PROBABILIDAD DE PARTICIPAR EN EL MERCADO LABORAL SEGÚN FORTALEZA DE LAZOS SOCIALES CON VECINOS. EFECTOS MARGINALES DE UN MODELO PROBIT

\begin{tabular}{|c|c|c|c|c|}
\hline \multirow[t]{2}{*}{$\begin{array}{l}\text { Variable dependiente: } \\
\text { 1: Participa en el mercado laboral } 2017\end{array}$} & \multicolumn{2}{|c|}{$\begin{array}{l}\text { Mujeres con lazos } \\
\text { débiles }\end{array}$} & \multicolumn{2}{|c|}{$\begin{array}{l}\text { Mujeres con lazos } \\
\text { fuertes }\end{array}$} \\
\hline & (1) & (2) & (3) & (4) \\
\hline \multirow[t]{2}{*}{ Tasa PLF zona censal 2017} & $0,084^{* *}$ & $0,089^{* *}$ & 0,013 & 0,011 \\
\hline & $(0,042)$ & $(0,040)$ & $(0,038)$ & $(0,039)$ \\
\hline \multirow[t]{2}{*}{ Proporción de mujeres en zona censal 2017} & 0,177 & 0,410 & $-3,850^{*}$ & $-3,907^{*}$ \\
\hline & $(2,716)$ & $(2,668)$ & $(2,090)$ & $(2,103)$ \\
\hline \multirow[t]{2}{*}{ Edad } & 0,013 & 0,013 & $0,056^{* * *}$ & $0,055^{* * *}$ \\
\hline & $(0,014)$ & $(0,014)$ & $(0,015)$ & $(0,015)$ \\
\hline \multirow[t]{2}{*}{ Edad2 } & $-0,000^{*}$ & $-0,000^{*}$ & $-0,001^{* * *}$ & $-0,001^{* * *}$ \\
\hline & $(0,000)$ & $(0,000)$ & $(0,000)$ & $(0,000)$ \\
\hline \multirow[t]{2}{*}{ 1:Tiene pareja } & $-0,001$ & $-0,004$ & $-0,127^{* *}$ & $-0,128^{* *}$ \\
\hline & $(0,064)$ & $(0,059)$ & $(0,062)$ & $(0,062)$ \\
\hline \multirow[t]{2}{*}{ Número de niños $<6$ años } & $-0,071$ & $-0,070$ & $-0,002$ & $-0,008$ \\
\hline & $(0,050)$ & $(0,050)$ & $(0,052)$ & $(0,051)$ \\
\hline \multirow[t]{2}{*}{ 1: Reside en RM } & 0,016 & 0,022 & $-0,075$ & $-0,077$ \\
\hline & $(0,075)$ & $(0,070)$ & $(0,076)$ & $(0,077)$ \\
\hline \multirow[t]{2}{*}{ 1: Es principal sostenedor del hogar } & 0,092 & $0,109^{*}$ & $0,164^{* * *}$ & $0,161^{* * *}$ \\
\hline & $(0,063)$ & $(0,060)$ & $(0,062)$ & $(0,062)$ \\
\hline \multirow[t]{2}{*}{ Log ingreso no laboral per cápita } & $-0,099 * *$ & $-0,095^{* *}$ & $-0,072^{* *}$ & $-0,073^{* *}$ \\
\hline & $(0,041)$ & $(0,041)$ & $(0,034)$ & $(0,034)$ \\
\hline \multirow[t]{2}{*}{ Calidez madre trabajadora con hijos ${ }^{\mathrm{a}}$} & & 0,025 & & 0,040 \\
\hline & & $(0,058)$ & & $(0,057)$ \\
\hline \multirow[t]{2}{*}{ Hombres y responsabilidades domésticas ${ }^{b}$} & & $-0,034$ & & 0,033 \\
\hline & & $(0,139)$ & & $(0,138)$ \\
\hline \multirow[t]{2}{*}{ Hombres y cuidado infantic ${ }^{c}$} & & 0,178 & & $-0,072$ \\
\hline & & $(0,163)$ & & $(0,183)$ \\
\hline Observaciones & 413 & 413 & 519 & 519 \\
\hline Media variable dependiente & 0,59 & 0,59 & 0,46 & 0,46 \\
\hline
\end{tabular}

Notas: Efectos marginales de la estimación de un modelo Probit en base a datos ELSOC (COES 2016, 2017) y CENSO 2017. PLF: Participación laboral femenina. a) De acuerdo con la afirmación: 'Una madre que trabaja puede establecer una relación tan cálida y sólida con sus hijos como una madre que no trabaja'; b) De acuerdo con la afirmación: 'Los hombres deberían asumir un mayor grado de responsabilidad en el trabajo doméstico que el que asumen actualmente', y c) De acuerdo con la afirmación: 'Los hombres deberían asumir un mayor grado de responsabilidad en el cuidado de los niños que el que asumen actualmente'. Mujeres con lazos débiles tienen un indicador de lazo social estimado menor a la mediana de 0,62. Errores robustos entre paréntesis. ${ }^{* * *} p<0,01 ;{ }^{* *} p<0,05 ;{ }^{*} p<0,1$.

Fuente: Elaboración propia en base a ELSOC (COES 2016, 2017) y CENSO 2017. 
y las especificaciones se diferencian solo por los controles incluidos, ya que la columna (2) incorpora las variables culturales. Igualmente, las columnas (3) y (4) muestran los resultados para las mujeres con lazos fuertes en el barrio.

Los resultados sugieren que la probabilidad individual de participar en el mercado laboral de las mujeres estaría asociada de manera positiva con la tasa de participación laboral de las vecinas que residen en la misma zona censal, solo para aquellas mujeres que cuentan con lazos débiles en el barrio. En efecto, el efecto marginal estimado es estadísticamente significativo para un nivel de significancia de $5 \%$. Esto sugiere que un aumento de una desviación estándar de la tasa de participación laboral femenina de las vecinas cercanas estaría asociado a un incremento en la probabilidad individual de participar de 8 puntos porcentuales, en promedio, y todo lo demás constante.

En contraste, la relación entre participación laboral individual y participación de las vecinas cercanas no se encuentra para el caso de mujeres que reportan contar con lazos fuertes dentro del barrio de residencia. El efecto marginal estimado resulta no ser estadísticamente significativo en las columnas (3) y (4) de la Tabla 2. Este resultado es consistente con lo señalado por Granovetter (1973), quien indicaba que los lazos débiles son relevantes para determinar comportamientos o resultados en el mercado laboral, porque generan puentes hacia información, grupos o contextos nuevos que los lazos más fuertes no son capaces de proveer al pertenecer todos a una misma red social.

Con respecto a las otras variables explicativas del modelo, se observa que para las mujeres con lazos débiles, el ser la principal sostenedora del hogar está asociado a un aumento de 10 puntos porcentuales en la probabilidad de participar en el mercado laboral, en comparación con las mujeres que no son la principal sostenedora. Asimismo, se puede observar que un aumento en el ingreso no laboral de las mujeres está asociado de manera negativa a la probabilidad de participar en el mercado del trabajo, con una disminución de 9 puntos porcentuales, en promedio, y todo lo demás constante.

Para el caso de las mujeres con lazos fuertes en el barrio, existen otras variables asociadas a la probabilidad de participar en el mercado laboral. En particular, si aumenta la proporción de mujeres en edad de trabajar en la zona censal, la probabilidad de participación disminuiría 3 veces, sugi- 
riendo un potencial efecto de competencia. El coeficiente estimado para la variable edad es estadísticamente significativo y positivo: un año más de la mujer se asocia a 5 puntos porcentuales más en la probabilidad de participar en el mercado del trabajo. Esta correlación crece a tasa decreciente, porque el cuadrado de la edad resulta tener un coeficiente estimado negativo y estadísticamente significativo. Tener pareja se relaciona de manera negativa, ya que la probabilidad de participar está asociada a 12 puntos porcentuales menos que aquellas que no tienen pareja. Finalmente, ser la principal sostenedora del hogar correlaciona de manera positiva con la probabilidad de participar, y lo contrario ocurre con un aumento en el ingreso no laboral del hogar per cápita, asociado a 7 puntos porcentuales menos en la probabilidad de participar en el mercado del trabajo.

\section{Conclusiones}

Este artículo analiza si existe relación entre las interacciones sociales de mujeres de baja escolaridad que ocurren en un mismo barrio y la decisión individual de cada mujer con respecto a participar o no en el mercado del trabajo. Además, se explora si el efecto es diferente para aquellas mujeres que poseen lazos fuertes y débiles con los vecinos.

Para comenzar, se desarrolla un modelo teórico que extiende el modelo clásico de oferta laboral femenina, incluyendo interacciones sociales. En particular, se introduce en la función de utilidad de cada mujer la tasa de participación de vecinas cercanas. De esta manera, cambios en las decisiones de las vecinas afectan el bienestar de cada mujer de manera individual, generándose una complementariedad estratégica. Es decir, cada mujer se ve beneficiada si las mujeres a su alrededor participan en el mercado laboral.

Para comprobar que este es el caso, se realiza una aplicación empírica del modelo. Para ello, con datos de las primeras dos rondas de ELSOC (COES 2016, 2017) y Censo 2017, se construye una base de datos inédita que cuenta, por un lado, con información demográfica y, por otro, con información territorial sobre la participación laboral de las mujeres que residen en una misma zona censal. Se estima, entonces, un modelo de participación laboral femenina. Esto es, a través de un modelo Probit, se estiman los factores asociados a la probabilidad de participar en el mercado del trabajo, incluyendo como variable explicativa la tasa de participación laboral de las mujeres residentes en el mismo barrio. 
Los resultados dan cuenta de la importancia de las interacciones y el tipo de lazos sociales conformados en el barrio para incrementar la inserción de mujeres de baja escolaridad en el mercado laboral de Chile.

Específicamente, las estimaciones sugieren que existiría una correlación positiva y estadísticamente positiva para aquellas mujeres de baja escolaridad que tienen lazos débiles con sus vecinos. En términos de magnitud, una mujer tendría 8 puntos porcentuales más de probabilidad de integrarse al mercado laboral si aumenta en una desviación estándar la proporción de mujeres del barrio que trabajan o buscan trabajo. Esta magnitud es similar a las estimaciones realizadas en otros países.

Los resultados son consistentes con la teoría de Granovetter (1973), quien señalaba la importancia de los lazos débiles para acceder a información a la cual no se tendría acceso si solo se interactuara con quienes se conforman lazos fuertes. Ello, porque los lazos débiles actuarían como puentes hacia otras redes sociales. Esto es particularmente importante en el contexto del mercado del trabajo, ya que los lazos débiles aportarían información sobre vacantes de empleo, que sería desconocida entre las personas con quienes se conforman lazos más fuertes.

Todo lo anterior tiene una implicancia relevante de política pública. Para incrementar la inserción de mujeres de baja escolaridad en el mercado laboral, resultaría beneficioso fomentar la interacción social entre vecinos a través de organizaciones comunales o a nivel de unidad vecinal. Generar instancias de colaboración entre vecinos y vecinas es beneficioso para que compartan información, especialmente en barrios de alta vulnerabilidad socioeconómica.

Asimismo, la presencia de estos efectos sociales puede ser utilizada por los hacedores de políticas para amplificar el efecto de programas a pequeña escala, que incentiven la participación de mujeres en el mercado laboral, en conjunto con instancias de interacción social a nivel de barrio que podrían generar efectos positivos sobre población no intervenida.

Finalmente, se debe señalar que los resultados presentados en este artículo son descriptivos y se debe avanzar hacia la estimación del efecto causal de las interacciones sociales sobre la participación laboral femenina en estudios futuros. No obstante, dada la escasa evidencia actualmente existente sobre este tema, este artículo establece las bases para una apasionante agenda de investigación que aborde los problemas de identificación provenientes de la simultaneidad de las decisiones de las 
mujeres y porque la distribución de las mujeres y sus características en el territorio no es aleatoria. La riqueza de la base de datos construida para el estudio que expone este artículo permite implementar métodos de identificación que están en la frontera de la literatura de interacciones sociales, tales como la utilización de variables instrumentales asociadas a características territoriales.

Asimismo, resultaría relevante analizar los mecanismos a través de los cuales se explicaría el efecto causal de las interacciones sociales, ya que podrían enriquecer las implicancias de política. Por ejemplo, si existiese un mecanismo de inspiración, entonces se podrían generar instancias donde jóvenes de baja escolaridad se involucren con la experiencia de mujeres similares que han participado en el mercado laboral, con trayectorias exitosas. De manera similar, si el mecanismo fuese la interdependencia de las restricciones presupuestarias, la provisión de servicios sociales, como mayor acceso a cuidado infantil en el vecindario, también tomaría relevancia. Estas reflexiones abren una nueva senda de investigación fascinante para explorar a futuro.

\section{Bibliografía}

Aizer, A. y Currie, J. 2004. Networks or Neighborhoods? Correlations in the Use of Publicly-Funded Maternity Care in California. Journal of Public Economics 88(12), 2573-2585. DOI: https://doi.org/10.1016/j.jpubeco.2003.09.003.

Akerlof, G.A. y Kranton, R.E. 2000. Economics and Identity. The Quarterly Journal of Economics 115(3), 715-753. DOl: https://doi.org/10.1162/003355300554881.

Andersson, R. y Musterd, S. 2005. Housing Mix, Social Mix, and Social Opportunities. Urban Affairs Review 40(6), 761-790. DOI: https://doi.org/10.1177/1078087405276006.

Andersson, R. y Musterd, S. 2010. What Scale Matters? Exploring the Relationships between Individuals' Social Position, Neighbourhood Context and the Scale of Neighbourhood. Geografiska Annaler: Series B, Human Geography 92(1), 23-43. DOI: https://doi.org/10.1111/j.1468-0467.2010.00331.x.

Atkinson, R., Dowling, R. y McGuirk, P. 2009. Home/Neighbourhood/City/+. Environment and Planning A 41, 2816-2822. DOI: https://doi.org/10.1068/a42110.

Bali Swain, R.B. y Wallentin, F.Y. 2012. Factors Empowering Women in Indian Self-Help Group Programs. International Review of Applied Economics 26(4), 425-444. DOI: https://doi.org/10.1080/02692171.2011.595398.

Bayer, P., Ross, S. y Topa, G. 2008. Place of Work and Place of Residence: Informal Hiring Networks and Labor Market Outcomes. Journal of Political Economy 116(6), 11501196. DOI: https://doi.org/10.1086/595975.

Behrman, J.R. y Wolfe, B.L. 1984. Labor Force Participation and Earnings Determinants for Women in the Special Conditions of Developing Countries. Journal of Development Economics 15(1-3), 259-288. DOI: https://doi.org/10.1016/03043878(84)90019-1. 
Bentancor, A. 2013. The Impact of Accessing Nursery Schools on Chilean Mothers Probability of Employment. Mimeo, Comunidad Mujer, julio. Disponible en: http://www.comunidadmujer.cl/biblioteca-publicaciones/2013/12/the-impactof-accessing-nursery-schools-on-chilean-mothers-probability-of-employment/ [15 de junio 2021].

Blundell, R. y Powell, J. 2004. Endogeneity in Semiparametric Binary Response Models. The Review of Economic Studies 71(3), 655-679. DOI: https://doi.org/10.1111/ j.1467-937X.2004.00299.x.

Blundell, R., Ham, J. y Meghir, C. 1987. Unemployment and Female Labour Supply. Economic Journal 97(388a), 44-64. DOl: https://doi.org/10.2307/3038229.

Boucher, V. y Goussé, M. 2019. Wage Dynamics and Peer Referrals. Review of Economic Dynamics 31, 1-23. DOI: https://doi.org/10.1016/j.red.2018.12.001.

Brock, W.A. y Durlauf, S.N. 2001a. Discrete Choice with Social Interactions. The Review of Economic Studies 68(2), 235-260. DOI: https://doi.org/10.1111/1467937X.00168.

Brock, W.A. y Durlauf, S.N. 2001b. Interactions-Based Models. Handbook of Econometrics 5, 3297-3380. DOI: https://doi.org/10.1016/S1573-4412(01)05007-3.

Brown, D.W. y Konrad, A.M. 2001. Granovetter Was Right: The Importance of Weak Ties to a Contemporary Job Search. Group \& Organization Management 26(4), 434462. DOI: https://doi.org/10.1177/1059601101264003.

Cahuc, P., Carcillo, S. y Zylberberg, A. 2014. Labor Economics. Cambridge, London: The MIT Press.

Carneiro, P., Galasso, E. y Ginja, R. 2013. The Impact of Providing Psycho-Social Support to Indigent Families and Increasing their Access to Social Services: Evaluating Chile Solidario. En Gender Impact: the World Bank's Gender Impact Evaluation Database. Washington DC: World Bank. Disponible en: http://documents. worldbank.org/curated/en/757521468236083208/The-impact-of-providingpsycho-social-support-to-indigent-families-and-increasing-their-access-tosocial-services-evaluating-Chile-Solidario [7 julio 2021].

Carneiro, P., Galasso, E. y Ginja, R. 2019. Tackling Social Exclusion: Evidence from Chile. The Economic Journal 129(617), 172-208. DOl: https://doi.org/10.1111/ ecoj.12594.

Casakin, H., Hernández, B. y Ruiz, C. 2015. Place Attachment and Place Identity in Israeli Cities: The Influence of City Size. Cities 42, 224-230. DOI: https://doi. org/10.1016/j.cities.2014.07.007.

CEPAL 2019. Coyuntura Laboral en América Latina y el Caribe: evolución y perspectivas de la participación laboral femenina en América Latina. Boletín CEPAL-OIT 21. Santiago: Comisión Económica para América Latina y el Caribe (CEPAL) y Organización Internacional del Trabajo (OIT) (LC/TS.2019/66). Disponible en: https://repositorio.cepal.org/handle/11362/44916 [22 de mayo 2021].

Chen, X. y Ge, S. 2018. Social Norms and Female Labor Force Participation in Urban China. Journal of Comparative Economics 46(4), 966-987. DOI: https://doi. org/10.1016/j.jce.2018.02.002.

Codazzi, K., Pero, V. y Albuquerque Sant'Anna, A. 2018. Social Norms and Female Labor Participation in Brazil. Review of Development Economics 22(4), 1513-1535. DOI: https://doi.org/10.1111/rode.12515.

COES 2016. Estudio Longitudinal Social de Chile. Primera Ola. [Archivo de datos.] Santiago: Centro de Estudios de Conflicto y Cohesión Social (COES). Disponible en: www.coes.cl [22 de mayo 2021]. 
COES 2017. Estudio longitudinal social de Chile. Segunda ola. [Archivo de datos.] Santiago: Centro de Estudios de Conflicto y Cohesión Social (COES). Disponible en: www.coes.cl [22 de mayo 2021].

Conley, T.G. y Topa, G. 2007. Estimating Dynamic Local Interactions Models. Journal of Econometrics 140(1), 282-303. DOI: https://doi.org/10.1016/j.jeconom.2006.09.012.

Contreras, D. y Plaza, G. 2010. Cultural Factors in Women's Labor Force Participation in Chile. Feminist Economics 16(2), 27-46. DOI: https://doi.org/10.1080/13545701003731815.

Contreras, D. y Sepúlveda, P. 2017. Effect of Lengthening the School Day on Mother's Labor Supply. The World Bank Economic Review 31(3), 747-766. DOI: https://doi. org/10.1093/wber/lhw003.

Contreras, D., Hurtado, A. y Sara, M. 2012. La excepción chilena y las percepciones de género en la participación laboral femenina. Serie de Documentos de Trabajo 372, Departamento de Economía, Universidad de Chile. Disponible en: http:// repositorio.uchile.cl/handle/2250/143740 [22 de mayo 2021].

Contreras, D., Otero, G., Díaz, J.D. y Suárez, N. 2019. Inequality in Social Capital in Chile: Assessing the Importance of Network Size and Contacts' Occupational Prestige on Status Attainment. Social Networks 58, 59-77. DOI: https://doi.org/10.1016/j. socnet.2019.02.002.

Duflo, E. 2012. Women Empowerment and Economic Development. Journal of Economic Literature 50(4), 1051-79. DOI: https://doi.org/10.1257/jel.50.4.1051.

Durlauf, S. 2004. Neighborhood Effects. Handbook of Regional and Urban Economics 4, 2173-2242. DOI: https://doi.org/10.1016/S1574-0080(04)80007-5.

Encina, J. y Martínez, C. 2009. Efecto de una mayor cobertura de salas cuna en la participación laboral femenina: evidencia de Chile. Serie Documentos de Trabajo 303, Departamento de Economía, Universidad de Chile. http://repositorio.uchile. cl/handle/2250/144070 [22 de mayo 2021].

Forrest, R. 2008. Who Cares about Neighbourhoods? International Social Science Journal 59(191), 129-141. DOI: https://doi.org/10.1111/j.1468-2451.2009.00685.x.

Forrest, R. y Kearns, A. 2001. Social Cohesion and Neighborhood Regeneration. Urban Studies 38, 2125-2143. DOI: https://doi.org/10.1080/00420980120087081.

García, C., Carrasco, J.A. y Rojas, C. 2014. El contexto urbano y las interacciones sociales: dualidad del espacio de actividades de sectores de ingresos altos y bajos en Concepción, Chile. Revista Latinoamericana de Estudios Urbano Regionales, EURE 40(121), 75-90. DOI: http://dx.doi.org/10.4067/S025071612014000300004.

Goldenberg, J. y Levy, M. 2009. Distance Is Not Dead: Social Interaction and Geographical Distance in the Internet Era. ArXiv preprint. Disponible en: https:// arxiv.org/abs/0906.3202 [22 de mayo 2021].

Goldin, C. 2006. The Quiet Revolution That Transformed Women's Employment, Education, and Family. American Economic Review 96(2), 1-21. DOI: https://doi.or g/10.1257/000282806777212350.

Granovetter, M. 1973. The Strength of Weak Ties. American Journal of Sociology 78(6), 1360-1380. DOI: https://doi.org/10.1086/225469.

Granovetter, M. 1983. The Strength of Weak Ties: A Network Theory Revisited. Sociological Theory 1, 201-233. DOI: https://doi.org/10.2307/202051.

Granovetter, M. 1995. Getting a Job: A Study of Contacts and Careers. Chicago: University of Chicago Press.

Greenberg, J. y Fernandez, R.M. 2016. The Strength of Weak Ties in MBA Job Search: A Within-Person Test. Sociological Science 3, 296-316. DOI: https://doi. org/10.15195/v3.a14. 
Guerra, J. y Mohnen, M. 2014. Occupational Choice and Social Interactions: A Study of Victorian London (17-89). Essays on Applied and Experimental Economics. Tesis Doctoral, University College London. En Guerra, J., Essays on Applied and Experimental Economics. Disponible en: https://discovery.ucl.ac.uk/id/ eprint/1456621/ [15 de junio 2021].

Guest, A.M. y Lee, B.A. 1983. The Social Organization of Local Areas. Urban Affairs Review 19(2), 217-240. DOI: https://doi.org/10.1177/004208168301900205.

Holanda, E. de 2000. Class Footprints in the Landscape. Urban Design International 5 , 189-198. DOI: https://doi.org/10.1057/palgrave.udi.9000015.

INE 2019. Antecedentes división político, administrativa y censal. Departamento de Geografía, Instituto Nacional de Estadísticas (INE). Disponible en: https:// geoarchivos.ine.cl/File/pub/presentaci\%C3\%B3n-dpa.pdf [22 de mayo 2021].

Kabeer, N. 2018. Gender, Livelihood Capabilities and Women's Economic Empowerment: Reviewing Evidence over the Life Course. Gender and Adolescence: Global Evidence (GAGE), London, UK. Disponible en: http://eprints.Ise. ac.uk/90462/ [22 de mayo 2021].

Kabeer, N. 2020. Women's Empowerment and Economic Development: A Feminist Critique of Storytelling Practices in 'Randomista' Economics. Feminist Economics 26(2), 1-26. DOI: https://doi.org/10.1080/13545701.2020.1743338.

Kearns, A. y Parkinson, M. 2001. The Significance of Neighbourhood. Urban Studies 38(12), 2103-2110. DOI: https://doi.org/10.1080/00420980120087063.

Killingsworth, M.R. y Heckman, J.J. 1986. Female Labor Supply: A Survey. Handbook of Labor Economics 1, 103-204. DOI: https://doi.org/10.1016/S1573-4463(86)01005-2.

Larrañaga, O. 2006. Participación laboral de la mujer en Chile: 1958-2003. En Valenzuela, J.S., Tironi, E. y Scully, T.R. (eds.), El eslabón perdido: familia, modernización y bienestar en Chile. Santiago: Taurus. Disponible en: https:// searchworks.stanford.edu/view/6520922 [22 de mayo 2021].

Lee, B.A. y Campbell, K.E. 1999. Neighbor Networks of Black and White Americans (119-146). En Wellman, B. y Boulder (eds.), Networks in the Global Village: Life in Contemporary Communities. Colorado: Westview Press. DOI: https://doi. org/10.4324/9780429498718.

LeSage, J.P. 2008. An Introduction to Spatial Econometrics. Revue d'économie industrielle (3), 19-44.

Link, F. y Valenzuela, F. 2018. La estructura de la densidad sociorresidencial en el área metropolitana de Santiago. Proyecto Fondecyt $N^{\circ} 1161550$. Instituto de Estudios Urbanos y Territoriales UC, Documentos de Trabajo del IEUT 3. Disponible en: https://estudiosurbanos.uc.cl/wp-content/uploads/2020/01/Doc_trabajo_redes. pdf [22 de mayo 2021].

Manski, C.F. 1993. Identification of Endogenous Social Effects: The Reflection Problem. The Review of Economic Studies 60(3), 531-542. DOI: https://doi. org/10.2307/2298123.

Manski, C.F. 2000. Economic Analysis of Social Interactions. Journal of Economic Perspectives 14(3), 115-136. DOI: https://doi.org/10.1257/jep.14.3.115.

Marques, E., Moya, E. y Zoppi, M. 2008. Personal Networks and Urban Poverty: Preliminary Findings. Brazilian Political Science Review 3, 10-34. DOI: https://doi. org/10.1257/jep.14.3.115.

Marsden, P.V. y Campbell, K.E. 1984. Measuring Tie Strength. Social Forces 63(2), 482501. DOI: https://doi.org/10.1093/sf/63.2.482. 
Martínez, C. y Perticará, M. 2017. Childcare Effects on Maternal Employment: Evidence from Chile. Journal of Development Economics 126, 127-137. DOI: https://doi. org/10.1016/j.jdeveco.2017.01.001.

Maurin, E. y Moschion, J. 2009. The Social Multiplier and Labor Market Participation of Mothers. American Economic Journal: Applied Economics 1(1), 251-272. DOI: https://doi.org/10.1257/app.1.1.251.

McKeown, S., Haji, R. y Ferguson, N. 2016. Understanding Peace and Conflict through Social Identity Theory: Contemporary Global Perspectives. Cham: Springer International Publishing.

MDS 2017. Síntesis de Resultados Casen 2017: Trabajo. Santiago: Ministerio de Desarrollo Social y Familia (MDS), Gobierno de Chile. Disponible en: http:// observatorio.ministeriodesarrollosocial.gob.cl/storage/docs/casen/2017/ Resultados_trabajo_Casen_2017.pdf https://estudiosurbanos.uc.cl/wp-content/ uploads/2020/01/Doc_trabajo_redes.pdf [22 de mayo 2021].

Medrano, P. 2009. Public Day Care and Female Labor Force Participation: Evidence from Chile. Serie Documentos de Trabajo 306, Departamento de Economía, Universidad de Chile. Disponible en: http://repositorio.uchile.cl/handle/2250/144031 https:// estudiosurbanos.uc.cl/wp-content/uploads/2020/01/Doc_trabajo_redes.pdf [22 de mayo 2021].

Méndez, M.L. y Otero, G. 2018. Neighbourhood Conflicts, Socio-Spatial Inequalities, and Residential Stigmatisation in Santiago, Chile. Cities 74, 75-82. DOI: https:// doi.org/10.1016/j.cities.2017.11.005.

Méndez, M.L., Otero, G., Link, F., López Morales, E. y Gayo, M. 2020. Neighbourhood Cohesion as a Form of Privilege. Urban Studies 58(8), 1691-1711. DOI: https://doi. org/10.1177/0042098020914549.

Mincer, J. 1962. Labor Force Participation of Married Women: A Study of Labor Supply (63-105). En Universities-National Bureau Committee for Economic Research (ed.), Aspects of Labor Economics. Princeton: Princeton University Press.

Montgomery, J.D. 1992. Job Search and Network Composition: Implications of the Strength-of-Weak-Ties Hypothesis. American Sociological Review 57(5), 586-596. DOI: https://doi.org/10.2307/2095914.

Moran, P. 1948. The Interpretation of Statistical Maps. Journal of the Royal Statistical Society Series B 10, 243-251. DOI: https://doi.org/10.1111/j.2517-6161.1948. tb00012.x.

Mota, N., Mae, F., Patacchini, E. y Rosenthal, S.S. 2016. Neighborhood Effects, Peer Classification, and the Decision of Women to Work. IZA Discussion Paper 9985. Disponible en: https://ssrn.com/abstract=2797556 [22 de mayo 2021].

Nicoletti, C., Salvanes, K.G. y Tominey, E. 2018. The Family Peer Effect on Mothers' Labor Supply. American Economic Journal: Applied Economics 10(3), 206-234. DOI: https://doi.org/10.1257/app.20160195.

Novy, A., Swiatek, D.C. y Moulaert, F. 2012. Social Cohesion: A Conceptual and Political Elucidation. Urban Studies 49(9), 1873-1889. DOI: https://doi. org/10.1177/0042098012444878.

OECD 2020. Labour Market Statistics: Labour Force Statistics by Sex and Age: Indicators, OECD Employment and Labour Market Statistics (database). DOI: https://doi.org/10.1787/data-00310-en.

Oreopoulos, P. 2003. The Long-Run Consequences of Living in a Poor Neighborhood. The Quarterly Journal of Economics 118(4), 1533-1575. DOI: https://doi. org/10.1162/003355303322552865. 
Patacchini, E., Picard, P.M. y Zenou, Y. 2015. Urban Social Structure, Social Capital and Spatial Proximity. CEPR Discussion Paper DP10501. Disponible en: https://ssrn. com/abstract $=2584018$.

Piras, C. y Rucci, G. 2014. Chile: participación laboral femenina y calidad del empleo. Banco Interamericano de Desarrollo. Nota Técnica 777. Disponible en: https:// publications.iadb.org/es/publicacion/13855/chile-participacion-laboralfemenina-y-calidad-del-empleo [22 de mayo 2021].

Ritzen, J., Easterly, W. y Woolcock, M.J. 2000. On 'Good' Politicians and 'Bad' Policies: Social Cohesion, Institutions, and Growth. Policy Research Working Paper 2448. World Bank. DOI: https://doi.org/10.1596/1813-9450-2448.

Rosenbaum, J.E., DeLuca, S., Miller, S.R. y Roy, K. 1999. Pathways into Work: Short-and Long-Term Effects of Personal and Institutional Ties. Sociology of Education 62(3), 179-196. DOI: https://doi.org/10.2307/2673228.

Schmutte, I.M. 2015. Job Referral Networks and the Determination of Earnings in Local Labor Markets. Journal of Labor Economics 33(1), 1-32. DOI: https://doi. org/10.1086/677389.

Soetevent, A.R. y Kooreman, P. 2007. A Discrete-Choice Model with Social Interactions: with an Application to High School Teen Behavior. Journal of Applied Econometrics 22(3), 599-624. Disponible en: https://doi.org/10.1002/jae.924.

Tapia, R., Lange, C. y Larenas, J. 2009. Factores de deterioro del hábitat residencial y de vulnerabilidad social en la conformación de barrios precarios. Breve revisión de algunos programas de barrios en Chile y en la región. Subprograma Domeyko política, pobreza y exclusión social. Vicerrectoría de Investigación y Desarrollo, Departamento de Investigación, Universidad de Chile. Cuaderno de Trabajo 1, 47-93. Disponible en: http://repositorio.uchile.cl/handle/2250/144796 [22 de mayo 2021].

Tapia, V. 2013. El concepto de barrio y el problema de su delimitación: Aportes de una aproximación cualitativa y etnográfica. Bifurcaciones 12. Disponible en: http:// www.bifurcaciones.cl/2013/03/el-concepto-de-barrio-y-el-problema-de-sudelimitacion/ [7 de julio 2021].

Tobler, W.R. 1976. Spatial Interaction Patterns. Journal of Environmental Systems 6, 271301. Disponible en: http://pure.iiasa.ac.at/id/eprint/241/ [22 de mayo 2021].

Topa, G. 2001. Social Interactions, Local Spillovers and Unemployment. The Review of Economic Studies 68(2), 261-295. DOI: https://doi.org/10.1111/1467-937X.00169.

Topa, G. 2011. Labor Markets and Referrals. Handbook of Social Economics 1, 11931221. DOI: https://doi.org/10.1016/B978-0-444-53707-2.00005-0.

Vandecasteele, L. y Fasang, A.E. 2020. Neighbourhoods, Networks and Unemployment: The Role of Neighbourhood Disadvantage and Local Networks in Taking up Work. Urban Studies 58(4), 696-714. DOl: https://doi.org/10.1177/0042098020925374.

Weinberg, B.A., Reagan, P.B. y Yankow, J.J. 2004. Do Neighborhoods Affect Hours Worked? Evidence from Longitudinal Data. Journal of Labor Economics 22(4), 891-924. DOI: https://doi.org/10.1086/423158.

Wellman, B. 1996. Are Personal Communities Local? A Dumptarian Reconsideration. Social Networks 18(4), 347-354. DOI: https://doi.org/10.1016/0378-8733(95)00282-0.

Wellman, B. 2001. Physical Place and Cyberplace: The Rise of Personalized Networking. International Journal of Urban and Regional Research 25(2), 227-252. DOI: https:// doi.org/10.1111/1468-2427.00309. 


\section{Anexos}

\section{Anexo I.Autocorrelación espacial de las tasas de participación laboral femenina}

Figura A1.1. AUTOCORRELACIÓN ESPACIAL DE LAS TASAS DE PARTICIPACIÓN LABORAL FEMENINA. REGIONES EN LA ZONA NORTE DEL PAÍS, AÑO 2017

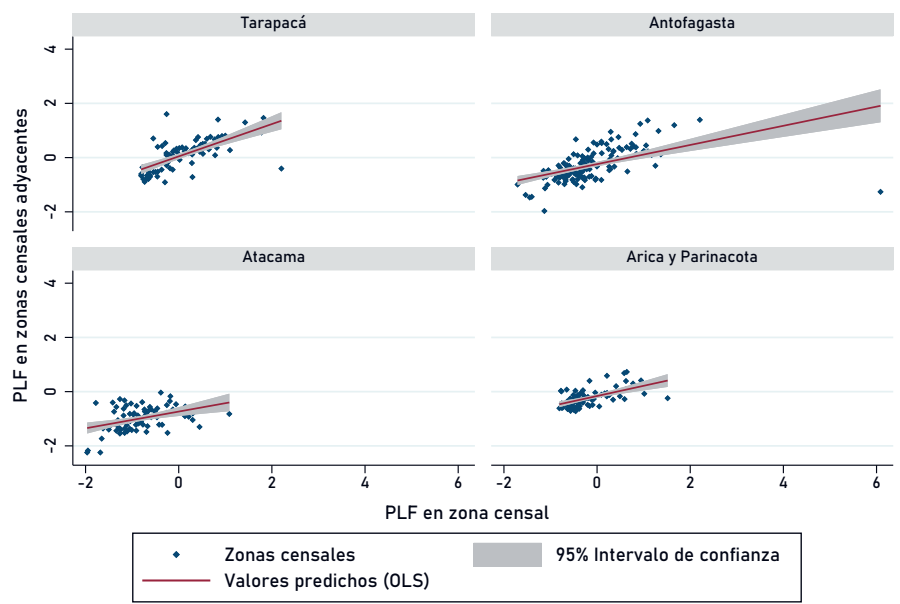

PLF: Participación Laboral Femenina (estandarizada)

Fuente: Elaboración propia en base a Censo 2017, INE.

Figura A1.2. AUTOCORRELACIÓN ESPACIAL DE LAS TASAS DE PARTICIPACIÓN LABORAL FEMENINA. REGIONES DE ALTA DENSIDAD EN LA ZONA CENTRO DEL PAÍS, AÑO 2017

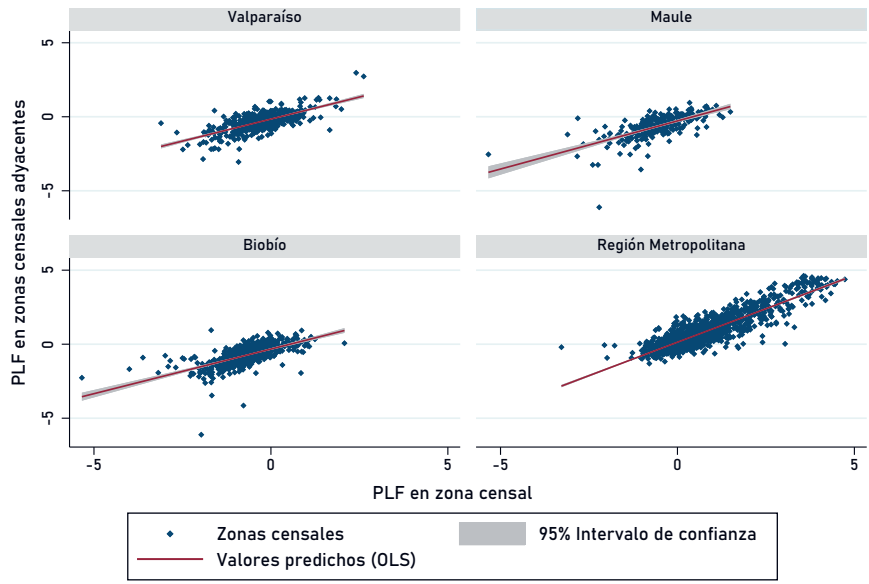

PLF: Participación Laboral Femenina (estandarizada)

Fuente: Elaboración propia en base a Censo 2017, INE. 
Figura A1.3. AUTOCORRELACIÓN ESPACIAL DE LAS TASAS DE PARTICIPACIÓN LABORAL FEMENINA. REGIONES DE MENOR DENSIDAD EN LA ZONA CENTRO DEL PAÍS, AÑO 2017

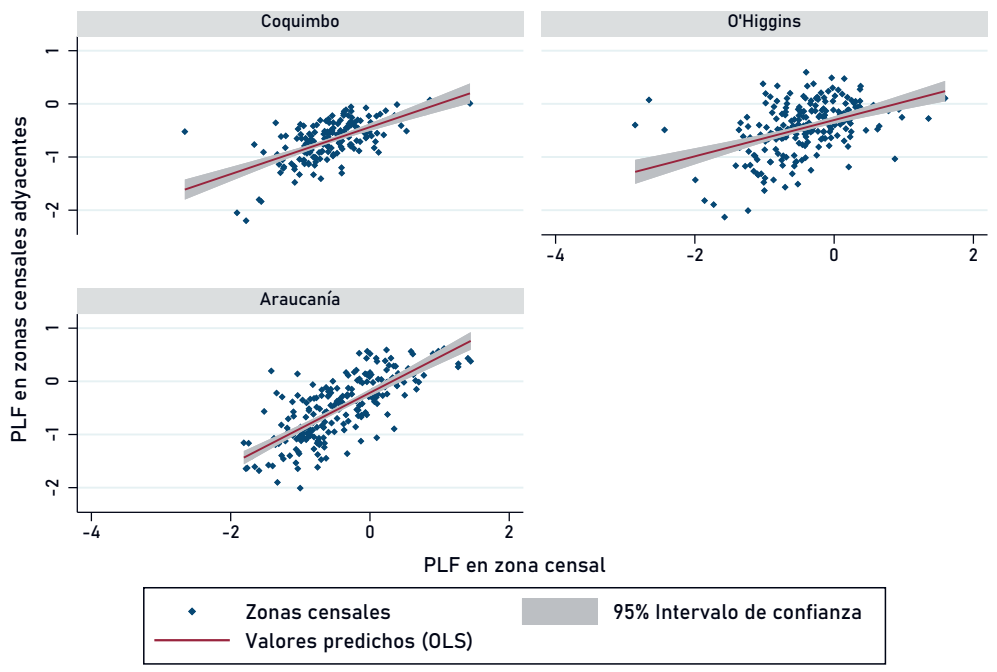

PLF: Participación Laboral Femenina (estandarizada)

Fuente: Elaboración propia en base a Censo 2017, INE.

Figura A1.4. AUTOCORRELACIÓN ESPACIAL DE LAS TASAS DE PARTICIPACIÓN LABORAL FEMENINA. REGIONES DE LA ZONA SUR DEL PAÍS, AÑO 2017

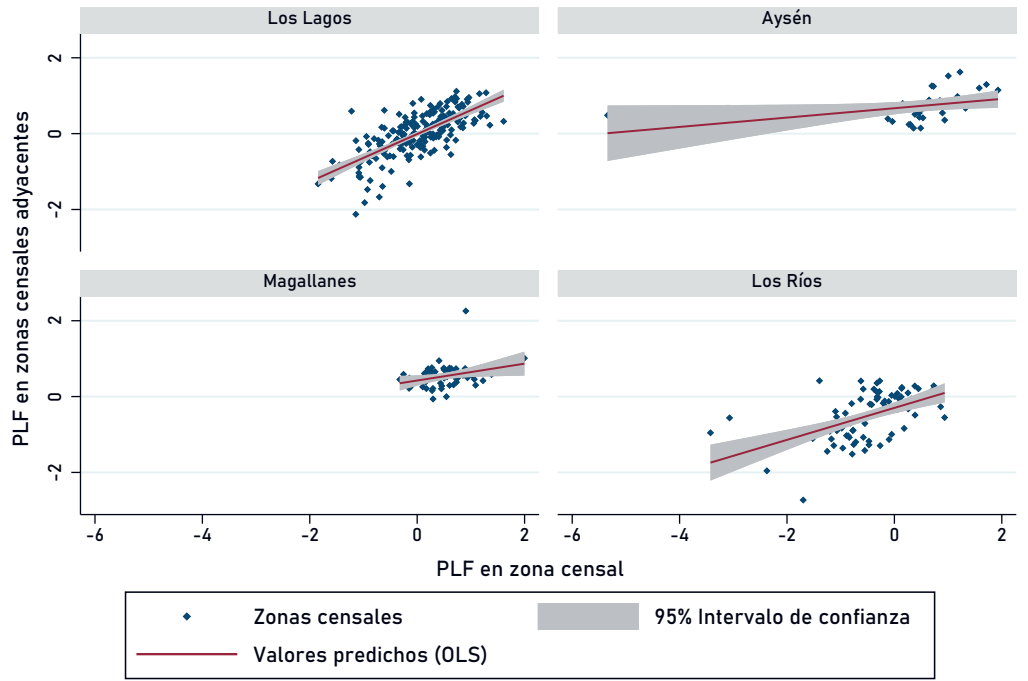

PLF: Participación Laboral Femenina (estandarizada)

Fuente: Elaboración propia en base a Censo 2017, INE. 


\section{Anexo 2. Significancia estadística del índice de autocorrelación espacial de Moran}

La significancia estadística del índice de Moran (1948) se extrae a partir de simulaciones de Monte Carlo. Específicamente, se realiza un test de permutación, el cual consiste en permutar aleatoriamente las tasas de participación laboral femenina entre las zonas censales y calcular el índice de Moran. Este proceso se realiza para cada iteración. La Figura A2.1 muestra la distribución de los indicadores de Moran obtenidos en una simulación con 200 permutaciones. Por construcción, la distribución se centra en cero, ya que los indicadores representan la correlación entre tasas de participación que existirían en un escenario de distribución aleatoria perfecta. La línea roja vertical indica el indicador de Moran observado igual a 0,89 . Por lo tanto, se infiere que este valor no es esperable si las tasas de participación se distribuyeran de manera aleatoria en el territorio.

En términos de significancia estadística, el pseudo $p$-value ${ }^{16}$ estimado es 0,005 . Esto sugiere que existe menos de un $1 \%$ de probabilidad de cometer error al rechazar la hipótesis nula de distribución aleatoria perfecta.

Figura A2.1. DISTRIBUCIÓN DEL INDICADOR DE MORAN DE AUTOCORRELACIÓN ESPACIAL A PARTIR DE 200 PERMUTACIONES. REGIÓN METROPOLITANA, AÑO 2017

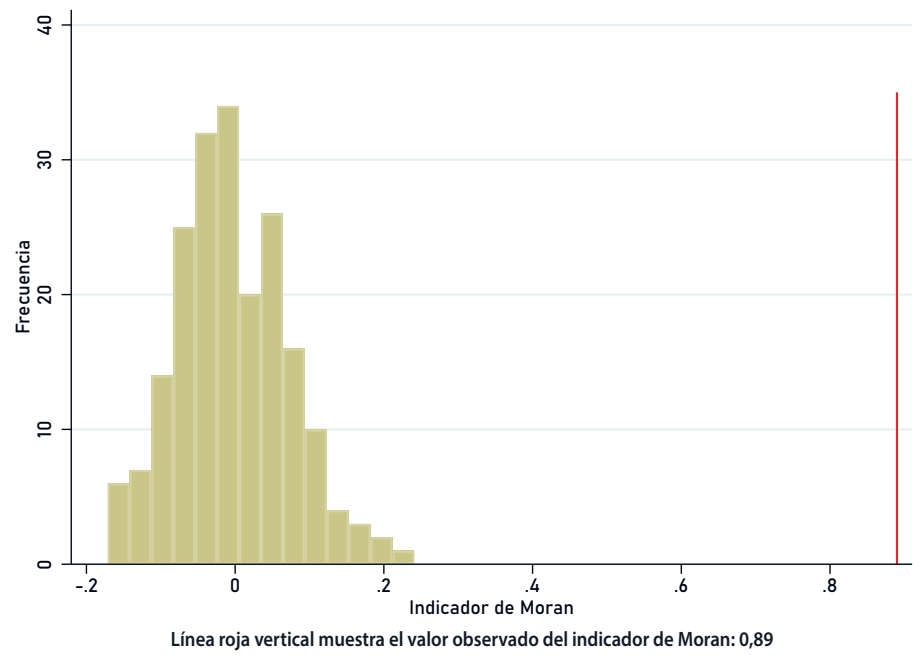

Fuente: Elaboración propia en base a Censo 2017, INE.

${ }_{16}$ Se calcula como $\frac{N_{\text {extremo }}-1}{N-1}$, donde $N$ corresponde al número de permutaciones y $N_{\text {extremo }}$ el número de veces en que el indicador de Moran resulta ser mayor al indicador de Moran observado (en este caso particular es cero). 


\section{Anexo 3. Construcción del indicador de fortaleza de lazos sociales}

La fortaleza de lazos sociales se puede definir como una combinación lineal entre la cantidad de tiempo compartido, la intensidad emocional, la intimidad y confianza mutua, y la reciprocidad de la relación (Granovetter 1973). Los datos de ELSOC permiten construir un indicador de la fortaleza de los lazos conformados entre las mujeres encuestadas y sus vecinos.

Específicamente, se utilizan preguntas de ELSOC que describen la relación entre cada mujer y sus vecinos, y están asociadas a cada dimensión (cantidad de tiempo, intensidad emocional, intimidad y reciprocidad). Luego, para cada pregunta se crea una variable binaria que toma el valor 1 si la respuesta de la mujer indica una alta o buena relación, 0 si la respuesta indica una baja relación con sus vecinos. A partir de las variables binarias, se calcula el promedio de cada dimensión. Por último, con estas cuatro variables se toma el promedio final para obtener el indicador final de fortaleza de lazos sociales conformados entre cada mujer y sus vecinos.

Primero, la dimensión tiempo hace referencia a la frecuencia de contacto que tiene la mujer con sus vecinos (Granovetter 1973). En esta dimensión se utiliza la pregunta: '¿Ha visitado la casa de algún vecino (en los últimos 12 meses)?', y se crea una variable denominada tiempo, que toma el valor 1 si 'Lo hizo 1 o 2 veces' o si 'Lo hizo más de dos veces', y 0 si 'Nunca lo hizo'.

Segundo, la dimensión intensidad es definida por Marsden y Campbell (1984) como la cercanía que tienen dos personas. Para esta dimensión se utilizan tres preguntas. La primera es: '¿En este barrio es fácil hacer amigos?'. A partir de ella se crea una variable binaria que toma el valor 1 si la respuesta es 'De acuerdo' o 'Totalmente de acuerdo', y 0 si la mujer responde 'Totalmente desacuerdo', 'En desacuerdo' o 'Ni de acuerdo ni en desacuerdo'. La segunda pregunta señala si la mujer encuestada ha recibido 'Amenazas, insultos $u$ ofensas de parte de vecinos de su barrio'. Para esta pregunta se crea una variable binaria que toma el valor 1 si responde 'Nunca', y 0 si responde 'Pocas veces', 'Algunas veces,' 'Muchas veces' o 'Siempre.' La tercera pregunta es: 'Mis vecinos y/o amigos se fueron o se están yendo del barrio', y se crea una variable que toma el valor 1 
si responde 'Totalmente en desacuerdo', 'En desacuerdo' o 'Ni de acuerdo ni en desacuerdo', y 0 si la encuestada está 'De acuerdo' o 'Totalmente de acuerdo.' Con las tres variables binarias, se calcula el promedio de la categoría para cada mujer, como una variable denominada intensidad.

Tercero, la dimensión intimidad se construye como un indicador de la variedad de temas tratados y de la confianza para hablar de temas personales entre individuos (Marsden y Campbell 1984). Para esta dimensión se utilizan tres preguntas. La primera es: '¿La gente en este barrio es sociable?', y se crea una variable binaria que toma el valor $1 \mathrm{si}$ la mujer responde 'De acuerdo' o 'Totalmente de acuerdo', y 0 si responde 'Totalmente en desacuerdo', 'En desacuerdo' o 'Ni de acuerdo ni en desacuerdo'. La segunda pregunta es: 'En términos generales, ¿cuánto confía usted en sus vecinos?'. Se crea una variable que toma el valor $1 \mathrm{si}$ la mujer responde 'Bastante' o 'Mucho', y 0 si responde 'Muy poco', 'Poco' o 'Algo'. La tercera pregunta es: ' $¿ \mathrm{H}$ Han llegado al barrio residentes que no le agradan?'. La variable binaria toma el valor 1 si la mujer responde 'Totalmente en desacuerdo', 'En desacuerdo' o'Ni de acuerdo ni en desacuerdo', y 0 si responde 'De acuerdo' o 'Totalmente de acuerdo'. Al igual que en la dimensión anterior, se toma un promedio de las tres variables binarias creadas, obteniendo la variable denominada intimidad.

La última dimensión es reciprocidad; consiste en los servicios mutuos y en la correspondencia de la relación entre cada mujer y sus vecinos. Para construir el indicador de esta dimensión, se utilizan dos preguntas. La primera es: '¿La gente en este barrio es colaboradora?'; se crea una variable binaria que toma el valor 1 si la mujer responde 'De acuerdo' o 'Totalmente de acuerdo', y 0 si responde 'Totalmente en desacuerdo', 'En desacuerdo' o 'Ni de acuerdo ni en desacuerdo'. La segunda pregunta es: '¿Aparecieron en el barrio actividades que no le agradan'. La variable binaria toma el valor 1 si la mujer responde 'Totalmente en desacuerdo', 'En desacuerdo' o 'Ni de acuerdo ni en desacuerdo', y 0 si responde 'De acuerdo' o 'Totalmente de acuerdo'. Luego se promedian las dos variables binarias para cada mujer, creando la variable denominada reciprocidad.

Finalmente, el indicador de fortaleza de lazos sociales corresponde al promedio entre las variables tiempo, intensidad, intimidad y reciprocidad. Este indicador varía entre 0 y 1 . Valores cercanos a 1 sugieren relaciones fuertes entre la mujer y sus vecinos. En contraste, si el indicador toma valores cercanos a 0 indicaría que la mujer tiene lazos débiles con sus vecinos. 


\section{Anexo 4. Spillovers proporcionales y complementariedad estratégica}

Siguiendo a Soetevent y Kooreman (2007), se puede comparar la utilidad entre escoger $y_{i g}=1$ o $y_{i g}=0$ cuando cualquier otra mujer $j$ escoge $y_{j g}=1$, y la utilidad entre escoger $y_{i g}=1 \circ y_{i g}=0$ cuando cualquier otra mujer $j$ escoge $y_{j g}=0$ :

$$
\begin{aligned}
& {\left[V\left(1, x_{i g}, 1, y_{-i j g}, \epsilon_{i g}(1)\right)-V\left(0, x_{i g}, 1, y_{-i j g}, \epsilon_{i g}(0)\right)\right] } \\
& -\left[V\left(1, x_{i g}, 0, y_{-i j g}, \epsilon_{i g}(1)\right)-V\left(0, x_{i g}, 0, y_{-i j g}, \epsilon_{i g}(0)\right)\right] \\
= & u\left(1, x_{i g}\right)+s\left(1,1, y_{-i j g}, x_{-i g}\right)+\epsilon_{i g}(1)-u\left(0, x_{i g}\right)-s\left(0,1, y_{-i j g}, x_{-i g}\right)-\epsilon_{i g}(0) \\
- & u\left(1, x_{i g}\right)-s\left(1,0, y_{-i j g}, x_{-i g}\right)-\epsilon_{i g}(1)+u\left(0, x_{i g}\right)+s\left(0,0, y_{-i j g}, x_{-i g}\right)+\epsilon_{i g}(0) \\
= & s\left(1,1, y_{-i j g}, x_{-i g}\right)-s\left(0,1, y_{-i j g}, x_{-i g}\right)-s\left(1,0, y_{-i j g}, x_{-i g}\right)+s\left(0,0, y_{-i j g}, x_{-i g}\right) \\
= & \frac{\gamma}{I-1}
\end{aligned}
$$

La expresión $y_{-i j g}$ indica las elecciones de todos los otros agentes, excluidos $i$ y $j$ del grupo $g$. 


\section{Anexo 5. Probabilidad de participar en el mercado laboral según género y educación}

Tabla A5.1. ESTIMACIÓN DE LA PROBABILIDAD DE PARTICIPAR EN EL MERCADO LABORAL SEGÚN GÉNERO Y NIVEL EDUCATIVO. EFECTOS MARGINALES DE UN MODELO PROBIT, AÑO 2017

\begin{tabular}{|c|c|c|c|c|c|c|}
\hline \multirow{3}{*}{$\begin{array}{l}\text { Variable dependiente: } \\
\text { 1: Participa en el mercado } \\
\text { laboral } 2017\end{array}$} & \multicolumn{3}{|c|}{ Hombres } & \multicolumn{3}{|c|}{ Mujeres } \\
\hline & Todos & $\begin{array}{l}\text { Educación } \\
\text { terciaria }\end{array}$ & $\begin{array}{l}\text { Educación } \\
\text { secundaria }\end{array}$ & Todas & $\begin{array}{l}\text { Educación } \\
\text { terciaria }\end{array}$ & $\begin{array}{l}\text { Educación } \\
\text { secundaria }\end{array}$ \\
\hline & (1) & (2) & (3) & (4) & (5) & (6) \\
\hline \multirow{2}{*}{$\begin{array}{l}\text { Tasa de participación laboral } \\
\text { en zona censal } 2017^{a}\end{array}$} & 0,012 & $-0,000$ & 0,028 & 0,011 & $-0,028$ & $0,054^{*}$ \\
\hline & $(0,016)$ & $(0,019)$ & $(0,020)$ & $(0,019)$ & $(0,023)$ & $(0,028)$ \\
\hline \multirow{2}{*}{$\begin{array}{l}\text { Proporción de hombres o } \\
\text { mujeres en zona censal } 2017\end{array}$} & 0,890 & 1,261 & 1,278 & $-1,320$ & $-1,352$ & $-2,103$ \\
\hline & $(1,135)$ & $(1,818)$ & $(0,964)$ & $(1,119)$ & $(1,511)$ & $(1,682)$ \\
\hline \multirow[t]{2}{*}{ Edad } & $0,020^{* * *}$ & $0,039 * * *$ & 0,008 & $0,054^{* * *}$ & $0,073^{* * *}$ & $0,033^{* * *}$ \\
\hline & $(0,005)$ & $(0,009)$ & $(0,005)$ & $(0,007)$ & $(0,008)$ & $(0,010)$ \\
\hline \multirow[t]{2}{*}{ Edad2 } & $-0,000^{* * *}$ & $-0,000^{* * *}$ & $-0,000 * *$ & $-0,001 * * *$ & $-0,001^{* * *}$ & $-0,000 * * *$ \\
\hline & $(0,000)$ & $(0,000)$ & $(0,000)$ & $(0,000)$ & $(0,000)$ & $(0,000)$ \\
\hline \multirow[t]{2}{*}{ 1:Tiene pareja } & 0,026 & $-0,078$ & $0,045^{*}$ & $-0,049$ & $-0,052$ & $-0,069$ \\
\hline & $(0,029)$ & $(0,065)$ & $(0,025)$ & $(0,036)$ & $(0,053)$ & $(0,048)$ \\
\hline \multirow[t]{2}{*}{ Número de niños $<6$ años } & 0,000 & 0,067 & $-0,040^{*}$ & $-0,010$ & $-0,029$ & $-0,042$ \\
\hline & $(0,028)$ & $(0,061)$ & $(0,023)$ & $(0,030)$ & $(0,036)$ & $(0,040)$ \\
\hline \multirow[t]{2}{*}{ 1: Reside en RM } & $-0,000$ & $-0,001$ & $-0,018$ & $-0,013$ & $-0,025$ & $-0,029$ \\
\hline & $(0,028)$ & $(0,041)$ & $(0,034)$ & $(0,040)$ & $(0,052)$ & $(0,055)$ \\
\hline \multirow{2}{*}{$\begin{array}{l}\text { 1: Es principal sostenedor del } \\
\text { hogar }\end{array}$} & $0,064^{* *}$ & 0,053 & $0,059^{* *}$ & $0,125^{* * *}$ & $0,100^{* *}$ & $0,135^{* * *}$ \\
\hline & $(0,032)$ & $(0,048)$ & $(0,029)$ & $(0,035)$ & $(0,050)$ & $(0,048)$ \\
\hline \multirow{2}{*}{$\begin{array}{l}\text { Log ingreso no laboral per } \\
\text { cápita }\end{array}$} & $-0,031^{* *}$ & $-0,036$ & $-0,042^{* * *}$ & $-0,071 * * *$ & $-0,045^{*}$ & $-0,091^{* * *}$ \\
\hline & $(0,015)$ & $(0,025)$ & $(0,014)$ & $(0,019)$ & $(0,025)$ & $(0,026)$ \\
\hline \multirow{2}{*}{ 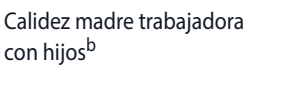 } & $-0,063^{* *}$ & $-0,092^{* *}$ & $-0,039^{*}$ & 0,016 & 0,014 & 0,029 \\
\hline & $(0,026)$ & $(0,043)$ & $(0,023)$ & $(0,033)$ & $(0,045)$ & $(0,044)$ \\
\hline \multirow{2}{*}{$\begin{array}{l}\text { Hombres y responsabilidades } \\
\text { domésticas }{ }^{c}\end{array}$} & $0,104^{* *}$ & $0,204^{* * *}$ & 0,008 & 0,043 & 0,136 & $-0,008$ \\
\hline & $(0,048)$ & $(0,074)$ & $(0,044)$ & $(0,086)$ & $(0,092)$ & $(0,113)$ \\
\hline \multirow[t]{2}{*}{ Hombres y cuidado infantil ${ }^{d}$} & 0,010 & $-0,086$ & 0,030 & 0,028 & $-0,065$ & 0,094 \\
\hline & $(0,056)$ & $(0,097)$ & $(0,054)$ & $(0,117)$ & $(0,134)$ & $(0,148)$ \\
\hline \multirow{2}{*}{$\begin{array}{l}\text { 1: Educación secundaria } \\
\text { completa o menos }\end{array}$} & $-0,011$ & & & $-0,165^{* * *}$ & & \\
\hline & $(0,028)$ & & & $(0,036)$ & & \\
\hline Observaciones & 845 & 304 & 541 & 1,342 & 410 & 932 \\
\hline $\begin{array}{l}\text { Promedio de la variable } \\
\text { dependiente }\end{array}$ & 0,86 & 0,86 & 0,87 & 0,59 & 0,71 & 0.52 \\
\hline
\end{tabular}

Notas: Efectos marginales de la estimación de un modelo Probit en base a datos ELSOC (COES 2016, 2017) y CENSO 2017. PLF: Participación laboral femenina. a) Corresponde a la tasa de participación laboral masculina en las regresiones para hombres y femenina en el caso de las mujeres; b) De acuerdo con la afirmación: 'Una madre que trabaja puede establecer una relación tan cálida y sólida con sus hijos como una madre que no trabaja'; c) De acuerdo con la afirmación: 'Los hombres deberían asumir un mayor grado de responsabilidad en el trabajo doméstico que el que asumen actualmente, y d) De acuerdo con la afirmación: 'Los hombres deberían asumir un mayor grado de responsabilidad en el cuidado de los niños que el que asumen actualmente'. Errores robustos entre paréntesis. ${ }^{* * *} p<0,01 ;{ }^{* *} p<0,05 ;{ }^{*} p<0,1$. Fuente: Elaboración propia en base a ELSOC (COES 2016, 2017) y CENSO 2017. 